\title{
System Identification and Distributed Control for Multi-rate Sampled Systems
}

\author{
Quan Min Shao ${ }^{\mathrm{a}, \mathrm{b}}$, Ali Cinar ${ }^{\mathrm{a}, *}$ \\ ${ }^{a}$ Department of Chemical and Biological Engineering, Illinois Institute of Technology, Chicago, IL 60616, \\ USA \\ ${ }^{b}$ Air Products and Chemicals, Allentown, PA 18195, USA
}

\begin{abstract}
System outputs with different sampling times may challenge traditional subspace identification methods to generate accurate process models and consequently provide model-based control systems that may not be very effective. The multi-rate identification problem is addressed by dividing the multi-rate sampled system into different subsystems, and a multirate distributed model predictive control technique is proposed to control such systems. The performance of the proposed method is evaluated and illustrated by modeling and controlling the Tennessee Eastman challenge problem.
\end{abstract}

Keywords: Multi-rate sampling, Subspace identification, Multi-rate distributed model predictive control, Agent-based systems, Tennessee Eastman Challenge Problem

\section{INTRODUCTION}

Chemical processes may have controlled variables with different sampling rates. Classical subspace identification to model a multi-rate sampled system at basic rate (the greatest common factor of different sampling rates) may yield poor prediction results for the variables with large sampling times. A better approach for identification of multi-rate systems is based on a lifted model, in which the system inputs and outputs with slower sampling rates are lifted to a basic (fastest) rate, which will generate larger dimensions of inputs and outputs for system models. There are two alternatives for using the lifted model in control system design. The lifted model can be used directly in subspace predictive control [1], or the lifted model can be converted to the basic-rate model which is then used in regular model predictive control (MPC) [2]. The limitation of the first approach is the increase in dimensionality of the model due to lifting, and the worst situation happens when some of the variables have much slower sampling rates compared to the fast rate. For the second approach, the inaccuracy of the lifted model may cause noticeable errors in the basic-rate model when extracting the basic-rate model from the lifted model.

${ }^{*}$ Corresponding Author: Phone: 1-312-567-3042, fax: 1-312-567-8874, cinar@iit.edu 
We propose a new approach to solve the control problem of multi-rate sampled systems that utilizes the lifted model, and leverages the advantages of distributed control techniques. The key is to let the local controllers communicate with each other and generate the optimized inputs sequences, which guarantee global stability and (sub)optimality, similar to the concepts of distributed MPC (DMPC) and feasible cooperative MPC (FCMPC) [3, 4, 5, 6]. Extension of DMPC to nonlinear systems is also an active research area, such as an effective nonlinear DMPC based on Lyapunov-based MPC, which follows similar information exchange mechanism as FC-MPC [7]. Interest in distributed MPC has increased in recent years and various novel techniques and implementations have been reported $[8,9,10,11,12,13,14,15,16,17,18,19,20]$. In literature, centralized MPC was utilized to deal with multi-rate sampling process in [21], as well as distributed MPC in $[22,23]$. Compared to the distributed control in $[22,23]$ which are based on Nash equilibrium, the proposed method in this paper focuses on global objective therefore it is approaching Pareto optimality.

In the proposed method for a multi-rate sampled systems, system outputs will be assigned to different subsystems based on their sampling times (in each subsystem, all the controlled variables have the same sampling time, but it is not necessary to have all the variables that have the same sampling time in a single subsystem). Then, only input lifting (to basic rate) is required when identifying the lifted model of one subsystem, with the outputs unchanged, which further reduces the dimensions of the subsystem models. For the model of a subsystem, the influences from neighbor subsystems also need to be considered in order to develop distributed control. Thus, system identification (SI) should also include the related inputs of neighbor subsystems as inputs to this subsystem. Subspace identification will be utilized to obtain the state space model for each subsystem. After the distributed models under different sampling times are available, DMPC is proposed to design the control system for the process with multi-rate sampling.

The Tennessee Eastman (TE) challenge problem is used to illustrate the proposed method [24]. In the TE process, concentration measurements are sampled every 6 or 15 minutes, while other measurements are sampled at higher frequency and could be assumed to be continuous variables. Several groups proposed various control methods for the TE process. Ricker and his coworkers reported control systems ranging from PI base control and decentralized control, to first-principles modeling and state estimation, optimization of operating conditions, to nonlinear model predictive control [25, 26, 27, 28]. Srinivas and Arkun reported an identification-control scheme that achieved very good results with a DMC-based MPC technique that uses the identified linear model [29]. Most researchers focused on MPC with state space models. One drawback is the use of too many PI controllers that sometimes reduces the MPC to play a coordinator/assistant role in control. Juricek and Larimore used subspace identification based on canonical variate analysis (CVA) for SI of the TE process [30]. However, their base control for stabilizing the process is not desirable for a control problem; the E feed is used to control the reactor level and thus eliminates one important MV for regulating the product concentration. They also state that the composition measurements in the TE process were omitted to avoid a multi-rate sampling problem, which subspace methods do not readily handle. Consequently, their SI approach would not be 
useful for concentration control of the TE process.

Distributed control and multi-rate sampling paradigm fits well in the framework of MADCABS (Monitoring, Analysis, Diagnosis, and Control with Agent-Based Systems), a software platform developed at IIT to provide a real-time supervision and control system for distributed and networked processes [31, 32, 33, 34, 35]. MADCABS is a multi-agent system to implement adaptive, decentralized, hierarchical supervision of process operations (Figure 1). The proposed DMPC and a coordinator agent [36] implemented in MADCABS enable multi-rate sampling and enhance the closed-loop control functionality of MADCABS.

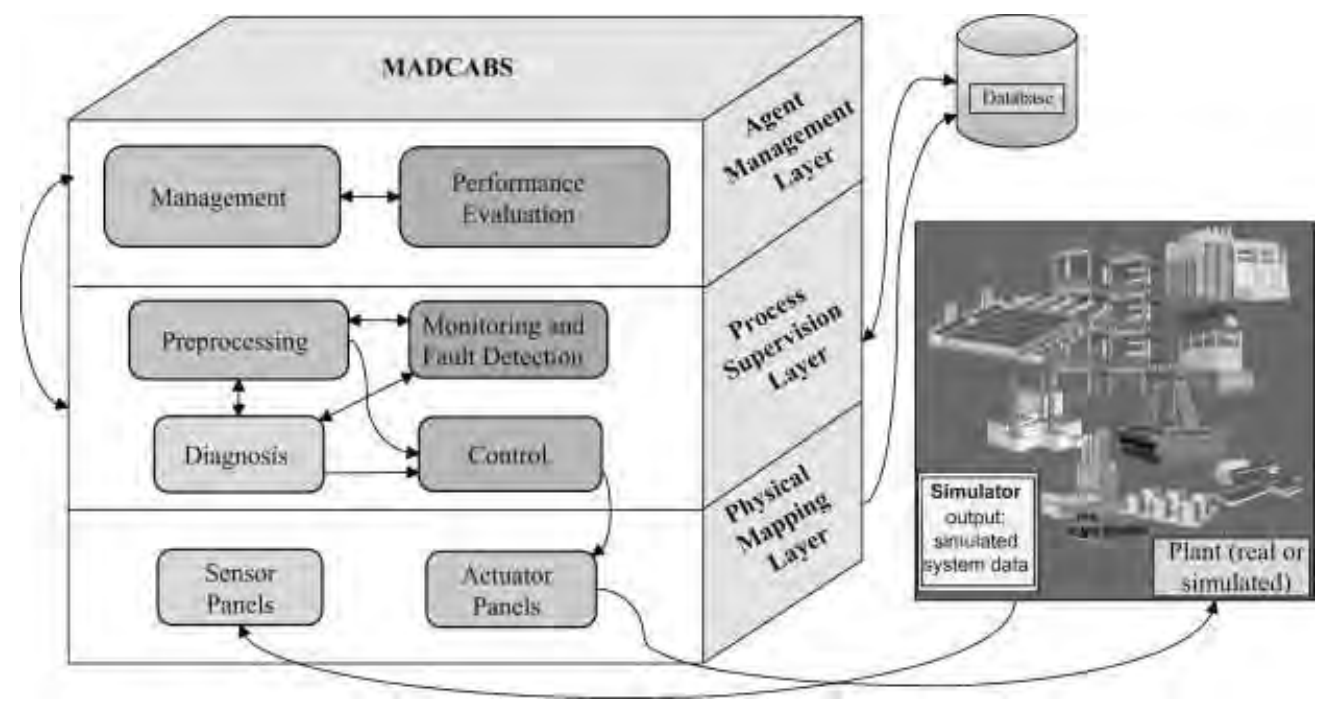

Figure 1: The framework of MADCABS.

The remainder of the paper is organized as follows. In Section 2, the motivation for the multi-rate system identification problem is illustrated by using the TE process. The distributed system identification method for multi-rate sampled systems is presented in Section 3, and multi-rate distributed model predictive control based on the identified model is proposed in Section 4. In Section 5, the results of multi-rate distributed control of the TE problem are given to illustrate the performance of the methods proposed. Conclusions are provided in Section 6.

\section{Motivation for use of the multi-rate identification}

Modeling of the TE challenge problem illustrates the limitations of using typical identification techniques for modeling multi-rate systems. When we used standard subspace identification $[37,38]$ to obtain the system model for MPC control of the TE challenge problem, the identified model yielded poor predictions for the controlled variables with larger sampling times and caused poor control. The main objective in the TE challenge problem is to maintain the product flowrate and composition at desired levels [24]. The process contains three main operation units: a reactor, a separator, and a stripper. It has total 41 measured variables and 12 manipulated variables (MV), four reactants labeled as A, C, D, and E, two 
products labeled as $\mathrm{G}$ and $\mathrm{H}$. Because of the process is unstable, base control consisting of several PI controllers is applied similar to [27]. First, the controlled variables (CV) and MVs are analyzed for defining the control problem. The MVs and CVs are the inputs and outputs for system identification, respectively. Since product concentration is directly related to the control objective, $\mathrm{G}$ concentration in product and the product flowrate are chosen as the $\mathrm{CV}$. Also, the reactor pressure is very sensitive to changes in process operations, and it may cause safety issues. Hence, it is selected as another CV. Several factors that affect the concentration of $\mathrm{G}$ in the product are considered as MVs. The feed $\mathrm{D}$ forms product $\mathrm{G}$, and $\mathrm{E}$ forms product $\mathrm{H}$ and thus will also influence the ratio of $\mathrm{G}$. Therefore, $\mathrm{D}$ and $\mathrm{E}$ feed are chosen as two MVs. Moreover, reactor pressure control is challenging because it is too sensitive to several variables, including $\mathrm{D}$ feed and $\mathrm{E}$ feed. When concentration control is sought, and D and E feeds change, the reactor pressure will be affected significantly. Initially, purge flowrate was picked as MV for the reactor pressure. This caused two problems and limited the ability to regulate reactor pressure. One problem is the dynamics between purge flowrate and reactor pressure that is not fast enough to cover dramatic changes in reactor pressure. The second problem is caused by the maximum purge flowrate which cannot compensate very large changes in reactor pressure. Thus, another MV (reactor temperature) is added to help reactor pressure control. The response of reactor pressure to changes in reactor temperature is fast, but large temperature changes could cause instability and this drawback must be dealt with carefully during control. The selected CVs and MVs are listed in Tables 1 and 2.

Pseudo random ternary sequence (PRTS) signals are sent to MVs to stimulate the system to generate data rich in dynamic variations for open-loop system identification. The amplitudes of the PRTS are listed in Table 2. The sampling time for TE process is 1 min (for variables with sampling time longer than $1 \mathrm{~min}$, the zero-order hold $(\mathrm{ZOH})$ is used, which means the measurement value is unchanged until the next update), and the switch time of PRTS is set to be $30 \mathrm{~min}$. In addition to the pure PRTS, some small white noise is also added to the PRTS to reduce the singularity of the intermediate matrices when performing system identification. The amplitude of white noise is set to be $10 \%$ of the amplitude of PRTS. The total sampling period is 96 hours [30].

Table 1: Notations of CVs for TE process

\begin{tabular}{cc}
\hline \hline Notation & CV \\
\hline$y_{1}$ & Product flowrate \\
$y_{2}$ & Product G concentration \\
$y_{3}$ & Reactor pressure \\
\hline
\end{tabular}

For validation, the responses of $\mathrm{CVs}$ to step changes in each $\mathrm{MV}$ are considered, and the amplitudes of the MVs are shown in the last column of Table 2. Three prediction horizons are considered and compared to assess the accuracy of the identified model in high, intermediate, and low frequencies in order to evaluate model performances for each data set:

- 1-step ahead prediction. The states are estimated by Kalman filter. 
- n-step ahead prediction. The states are estimated by using the estimated state in previous n-1 steps, since no measurement is available for the states, and a Kalman filter is utilized to compute the estimates of the states. Then, the identified model is used $n$ times to predict current states from the states n-steps ago. Note that the error terms are unknown when $\hat{x}(k \mid k-n)$ is predicted, thus they are all set to 0 . In this study $n=60$, which corresponds to 1 hour prediction.

- $\infty$-step ahead prediction. Start with the initial states, and update new states at every step by identified model, in which the error terms are all set to 0 .

Table 2: Notations of MVs for TE process, and the amplitudes of PRTS and step changes for system identification and validation respectively.

\begin{tabular}{cccc}
\hline \hline Notation & MV & $\delta u(\mathrm{SI})$ & $\delta u(\mathrm{Val})$ \\
\hline$u_{1}$ & D feed & $200 \mathrm{~kg} / \mathrm{s}$ & $80 \mathrm{~kg} / \mathrm{h}$ \\
$u_{2}$ & E feed & $200 \mathrm{~kg} / \mathrm{h}$ & $120 \mathrm{~kg} / \mathrm{h}$ \\
$u_{3}$ & Reactor temp. & $0.5{ }^{\circ} \mathrm{C}$ & $2{ }^{\circ} \mathrm{C}$ \\
$u_{4}$ & Purge flowrate & $0.05 \mathrm{kscmh}$ & $0.1 \mathrm{kscmh}$ \\
\hline
\end{tabular}

Next, all the CVs and MVs are used to develop an overall state space model. The 1-step ahead prediction almost overlaps with the original data for all validation results in this work, therefore the 1-step ahead prediction is be shown in the validation figures. The 60-step and $\infty$-step ahead predicted values for validation data are shown in Figure 2. The prediction of product flowrate is good for all cases; however, the change of flowrate is small, which means it is not sensitive to all controlled variables, and this causes trouble when implementing control. Steady state response of $\mathrm{G}$ concentration reacts well to changes in feed D (the most important $\mathrm{CV}$ for the control of $\mathrm{G}$ concentration in product), but its prediction in response to variations in feed $\mathrm{E}$ has large offsets, and its prediction for the other two inputs are very poor for the the validation data. This indicates that it is difficult for subspace identification to handle processes with multi-rate sampling. Furthermore, in practice if the correlation between an input and an output is small, which may cause a small signal-to-noise ratio, it is difficult to obtain a good model between them. The steady state offset is unavoidable due to the nonlinearity in predictions of pressure, and pressure control should be dealt with carefully.

These observations indicate that an approach that uses a single overall model to identify the multi-rate sampled system would fail to generate an accurate model for the CV with longer sampling times. Therefore, a more sophisticated model and its corresponding control algorithm are needed to identify and control such systems.

\section{Distributed Multi-rate System Identification}

In order to obtain a more accurate model for multi-rate sampled system, a distributed multi-rate system identification is proposed based on linear distributed state space model and lifted model. 

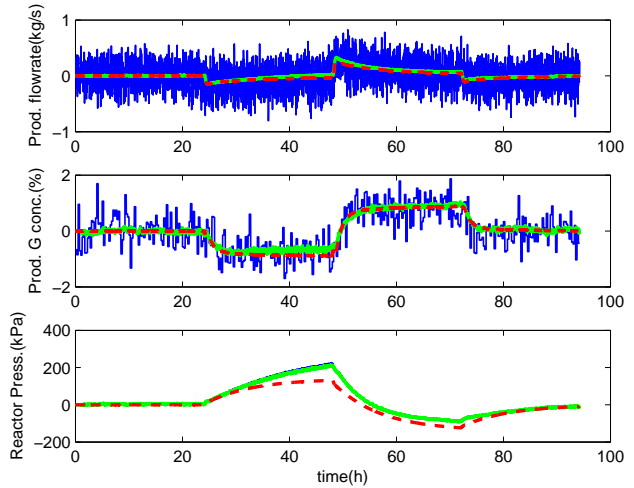

(a) Prediction results for step change of D feed.
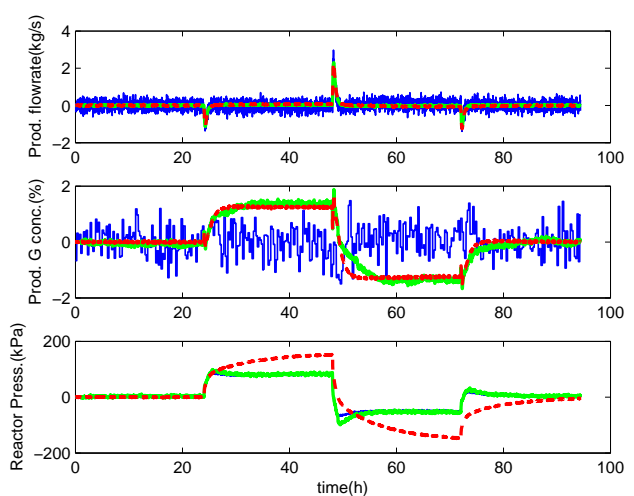

(c) Prediction results for step change of reactor temperature.
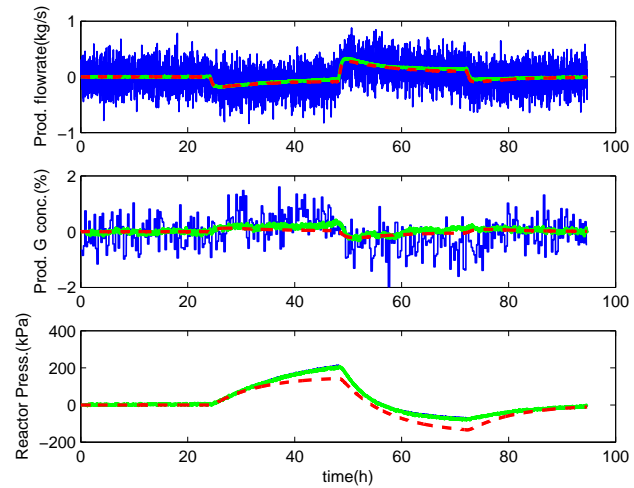

(b) Prediction results for step change of $\mathrm{E}$ feed.
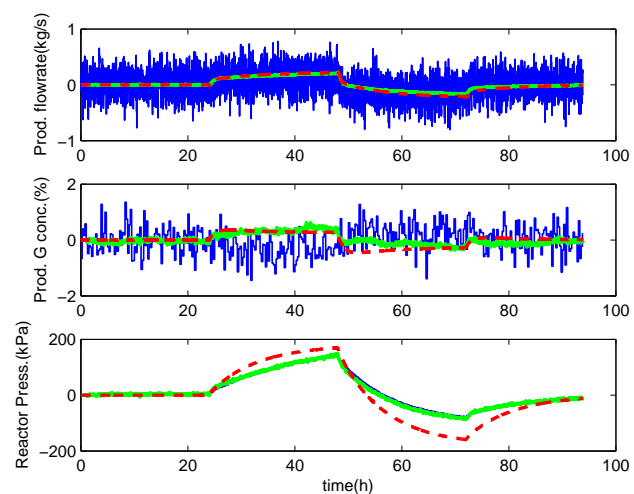

(d) Prediction results for step change of purge flowrate.

Figure 2: Overall model performance for 60-step and $\infty$-step ahead predictions for validation data. The blue line shows the original data, green bold (grey bold in black/white) and red dashed bold (black dashed bold in black/white) lines are the 60 -step and $\infty$-step ahead prediction results respectively. 


\subsection{Formulation of Distributed Multi-rate System Identification}

Consider a linear system described by the state space model

$$
\begin{aligned}
x(k+1) & =A x(k)+B u(k) \\
y(k) & =C x(k)
\end{aligned}
$$

The whole system can be divided to subsystems for distributed control, and each subsystem includes the inputs from other subsystems. The linear model for subsystem $i$ is

$$
\begin{aligned}
x_{i}(k+1) & =A_{i} x_{i}(k)+B_{i} u_{i}(k)+\sum_{j \neq i}^{M} W_{i j} u_{j i}(k) \\
y_{i}(k) & =C_{i} x_{i}(k)
\end{aligned}
$$

where $M$ is the number of subsystems, $u_{j i}$ is a subset of $u_{j}$ (the inputs in subsystem $j$ ) and it includes all the inputs that would affect $y_{i}$ (outputs in subsystem $i$ ) in $u_{j}$, and $W_{i j}$ is the corresponding input matrix of $u_{j i}$.

Standard subspace identification technique can be applied to obtain the above distributed model, by letting

$$
\begin{aligned}
\beta_{i} & =\left[B_{i}, \overline{B_{i}}\right]=\left[B_{i}, W_{i 1}, \ldots, W_{i(i-1)}, W_{i(i+1)}, \ldots, W_{i N}\right] \\
\mu_{i} & =\left[u_{i}^{T}, \bar{u}_{i}^{T}\right]^{T}=\left[u_{i}^{T}, u_{1 i}^{T}, \ldots, u_{(i-1) i}^{T}, u_{(i+1) i}^{T}, \ldots, u_{N i}^{T}\right]^{T}
\end{aligned}
$$

where ${\overline{u_{i}}}^{T}$ is the inputs from other subsystems to subsystem $i$, and $\overline{B_{i}}$ includes the corresponding input matrices. Then Equation 2 becomes

$$
x_{i}(k+1)=A_{i} x_{i}(k)+\beta_{i} \mu_{i}(k)
$$

For expressing the multi-rate sampled system, consider a system with a single sampling rate $T$, and a subsystem containing the controlled variables with sampling time $n T$. Using the format of the single-rate state space model described in Equation 1, the lifted model is:

$$
\begin{aligned}
x(k+n) & =\underline{A} x(k)+\underline{B} \underline{u}(k) \\
y(k) & =C x(k)
\end{aligned}
$$

where the underline indicates the lifted form and

$$
\begin{aligned}
\underline{A} & =A^{n} \\
\underline{B} & =\left[\begin{array}{llll}
A^{n-1} B & A^{n-2} B & \ldots & B
\end{array}\right] \\
\underline{u}(k) & =\left[\begin{array}{llll}
u(k)^{T} & u(k+1)^{T} & \ldots & u(k+n-1)^{T}
\end{array}\right]^{T}
\end{aligned}
$$

The lifted model describes the relation between the current state and the state after $n T$, which is the sampling time of the controlled variables. It considers all the inputs between two consecutive sampling times at time interval of $T$, which is the single rate of the whole discrete-time system. 
Then the lifted model for distributed model described in Equation 2 is:

$$
\begin{aligned}
x_{i}\left(k+n_{i}\right) & =A_{i}^{n_{i}} x_{i}(i)+\underline{B_{i}} \underline{u_{i}}(k)+\sum_{j \neq i}^{M} \underline{W_{i j}} \underline{u_{j i}}(k) \\
y_{i}(k) & =C_{i} x_{i}(k)
\end{aligned}
$$

where $W_{i j}$ is the lifted form of $W_{i j}$, which is analogous to $\underline{B}$ in Equation 6 .

The model for each subsystem can be written in the form similar to Equation 4:

$$
x_{i}\left(k+n_{i}\right)=\underline{A_{i}} x_{i}(k)+\underline{\beta_{i}} \underline{\mu_{i}(k)}
$$

where the system matrices and inputs are in the lifted form. Again, the above model (Equation 8) can be identified by the procedure of standard subspace identification.

\subsection{Results of Distributed Multi-rate System Identification}

For TE process, we partitioned the system to 2 subsystems in order to get more accurate models for control. The CVs are divided to two groups according to their sampling times. Product flowrate and reactor pressure are in subsystem 1, and $\mathrm{G}$ concentration in product is in subsystem 2. The MVs are also allocated to these two subsystems. D feed is in subsystem 2 because it is the main MV that controls $\mathrm{G}$ concentration in product, and the other $3 \mathrm{MVs}$ are in subsystem 1 (Figure 3).

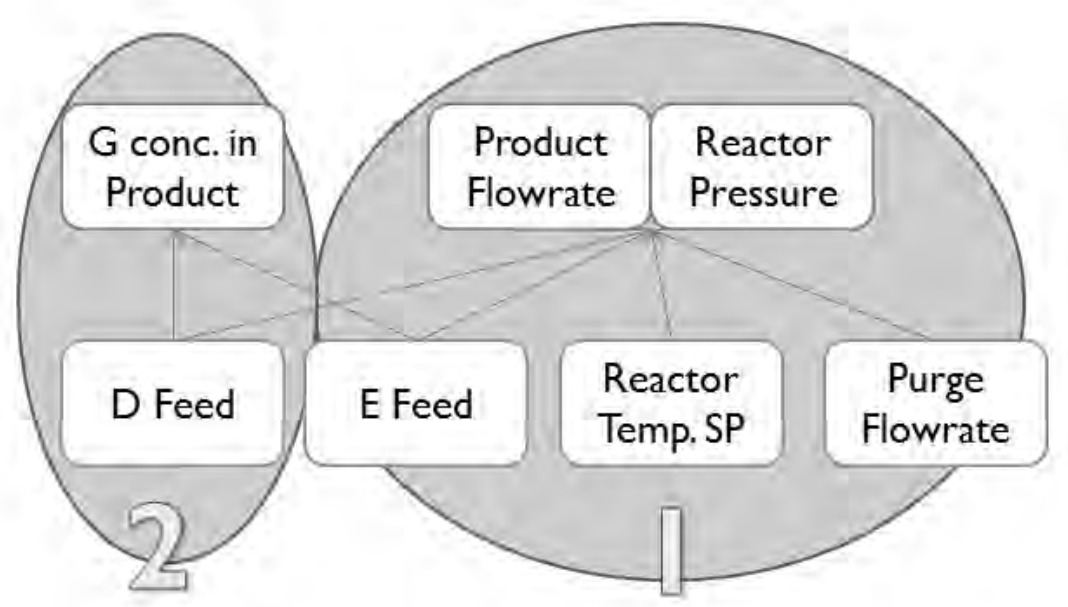

Figure 3: Distributed system partition.

For subsystem 1, the identification is straightforward, and since all $4 \mathrm{MVs}$ have significant influence on reactor pressure, and the $\mathrm{CVs}$ in subsystem 1 have single-rate sampling (they are sampled continuously at high frequency, so the sampling time can be set arbitrarily), standard subspace identification can be applied. First, the lifted model should be identified since it is a multi-rate sampling system. The total simulation period is 96 hour $=5760 \mathrm{~min}$, 
which means there are 5760 sampling points for a sampling time of 1 min. Identification is more challenging for subsystem 2 for several reasons. The sampling time is $15 \mathrm{~min}$ for $\mathrm{G}$ concentration in product, and only 384 sampling points are available for identification, and this many not be enough. Moreover, reactor temperature and purge flowrate have no significant influence on product $G$ concentration, thus it is not a good idea to include them as MVs in subsystem 2 for the large signal-noise ratio. The alternative solution is to make another simulation and increase the simulation time, and do not stimulate reactor temperature and purge flowrate. Note that it is not required to conduct another simulation (or test in a process) for the identification of distributed multi-rate system, as long as enough data are available to identify the model.

The validation responses are shown in Figure 4. Although models of subsystem 1 and 2 are identified separately, the results are displayed in the same figure at the same order of overall model case for comparison. The results for almost all the MVs have certain improvements. For subsystem 1, the same data set and same identification parameters are used, but because the multi-rate sampling CV is removed, the identified model accuracy

is improved. For subsystem 2, the identified lifted model is able to handle the multi-rate sampling variable more precisely to the step change of E feed.

\section{Multi-rate Distributed Control}

With the identified local model (either normal state space model or lifted model) for each subsystem, distributed control allows each local controller to optimize its own objective function and determine its own control actions, while the communications and iterations between local controllers ensure a suboptimal solution of the global objective function and also guarantee the stability of the control system.

\subsection{Multi-rate Model Predictive Control}

Consider the use of lifted model on a multi-rate centralized system, in which the sampling time of the controlled variables are integer times of basic rate, and its controller is assumed to update at basic-rate. Although this makes no sense in terms of improving the control performance, it will provide a baseline for assessing the performance of the distributed case.

For regular linear MPC with quadratic objective function:

$$
\begin{array}{r}
\Phi(k)=\sum_{t=k}^{k+N-1}\left(y(t \mid k)^{T} Q y(t \mid k)+u(t \mid k)^{T} R u(t \mid k)\right) \\
+y(k+N \mid k)^{T} \overline{Q_{f}} y(k+N \mid k)
\end{array}
$$

the objective function could be converted to a quadratic form under the constraints of Equation 1 as [39]:

$$
\Phi(k)=\left(u^{N}(k)\right)^{T} H u^{N}(k)+2\left(u^{N}(k)\right)^{T} G x(k)
$$

The first challenge for the multi-rate system would be the selection of an appropriate prediction horizon for the lifted model. At time $k$, the prediction horizon should include all 

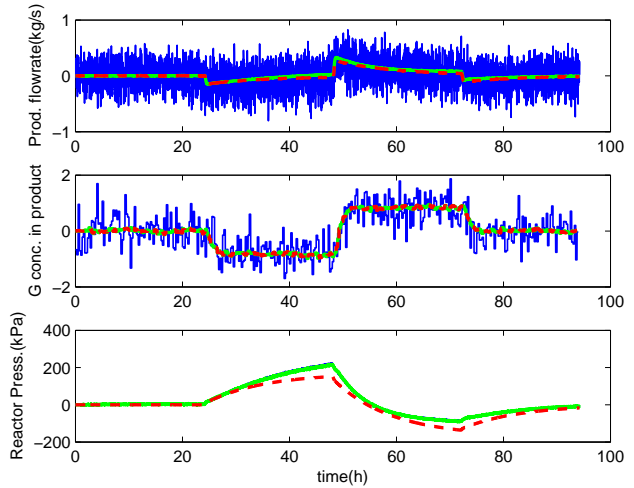

(a) Prediction results for step change in D feed.
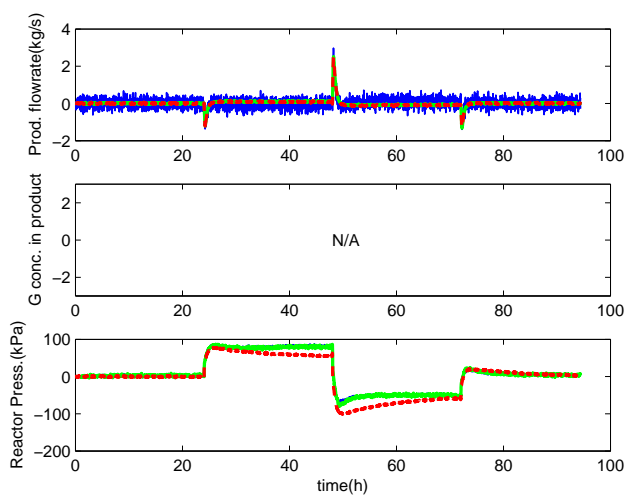

(c) Prediction results for step change in reactor temperature.
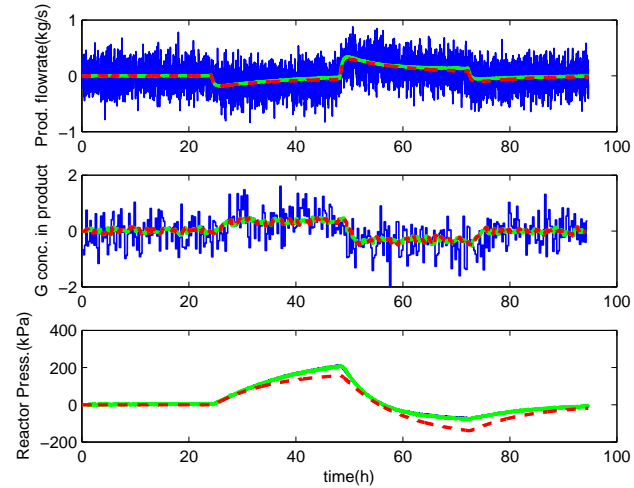

(b) Prediction results for step change in E feed.
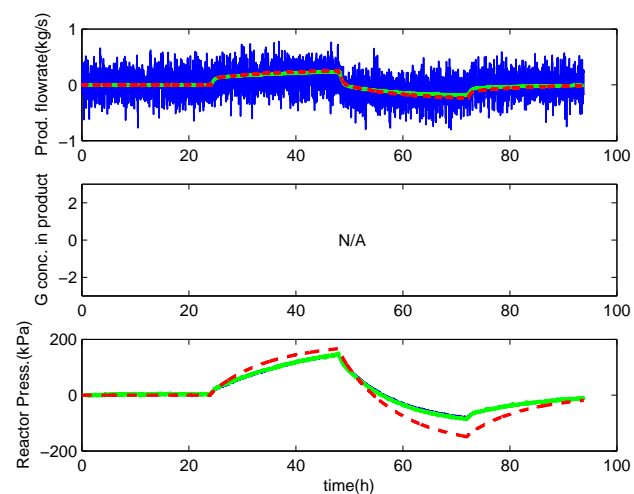

(d) Prediction results for step change in purge flowrate.

Figure 4: Distributed model performance for 60-step and $\infty$-step ahead predictions for validation data. The blue line shows the original data, green bold (grey bold in black/white) and red dashed bold (black dashed bold in black/white) lines are the 60 -step and $\infty$-step ahead prediction results respectively. 
sampling times in the range $k$ to $k+N-1$. The prediction horizon is determined by $N^{m}=\lfloor(N-2) / n+2\rfloor$, where $\lfloor x\rfloor$ denotes integer less than or equal to $x$. The single rate prediction horizon $N$ does not have to be the multiples of local sampling rates, and it was chosen such that its time frame is able to cover the dynamic of all the subsystems.

Assuming that the first sampling time is at $k_{0}$, define $s(k)=\left\lfloor\left(\left(k-k_{0}\right) / n\right)\right\rfloor * n+k_{0}$, which stands for the sampling time of current value. Then, using prediction horizon $N$ for regular $\mathrm{MPC}$, the objective function at $s(k)$ would be:

$$
\begin{aligned}
\Phi^{s}(s(k))= & \sum_{j=0}^{N^{m}-1}\left(y(k+j n \mid k)^{T} Q^{m} y(k+j n \mid k)\right. \\
& \left.+\underline{u}(k+j n \mid k)^{T} R^{m} \underline{u}(k+j n \mid k)\right) \\
& +y\left(k+N^{m} n \mid k\right)^{T} \bar{Q}_{f}^{m} y\left(k+N^{m} n \mid k\right) \\
= & \left(u^{N^{m} n}\right)(s(k))^{T} H^{m} u^{N^{m} n}(s(k)) \\
& +2\left(u^{N^{m} n}(s(k))\right)^{T} G^{m} x(s(k))
\end{aligned}
$$

where the superscript $s$ denotes sampling time, and $Q^{m}, R^{m}$, and $\overline{Q_{f}^{m}}$ are the penalty matrices for outputs, inputs, and terminal outputs, respectively, for the multi-rate system described by the lifted model. $u^{N^{m} n}$ is the input sequence with the length of $N^{m} n$.

Conduct the following partition:

$$
\begin{aligned}
H^{m} & =\left[\begin{array}{lll}
H_{11}^{m} & H_{12}^{m} & H_{13}^{m} \\
H_{21}^{m} & H_{22}^{m} & H_{23}^{m} \\
H_{31}^{m} & H_{32}^{m} & H_{33}^{m}
\end{array}\right] \\
G^{m} & =\left[\begin{array}{l}
H_{1}^{m} \\
H_{2}^{m} \\
H_{3}^{m}
\end{array}\right] \\
u^{N^{m} n} & =\left[\begin{array}{l}
u_{1}^{N^{m}} n \\
u_{2}^{N^{m} n} \\
u_{3}^{N^{m}} n
\end{array}\right]
\end{aligned}
$$

where $u_{1}^{N^{m} n}=[u(s(k)), \cdots, u(k-1)], u_{2}^{N^{m} n}=[u(k), \cdots$, $u(k+N-1)]$, and $u_{3}^{N^{m} n}=\left[u(k+N), \cdots, u\left(s(k)+N^{m} n-1\right)\right] . u_{1}^{N^{m} n}$ contains inputs from the most recent sampling time to the current time (not included), $u_{2}^{N^{m} n}$ contains inputs that start at the current time (included) and last for the time of control horizon, and $u_{3}^{N^{m} n}$ contains the rest of the inputs. The decomposition of $H^{m}$ and $G^{m}$ corresponds to the partition of $u^{N^{m} n}$. At time $k, u_{1}^{N^{m} n}$ is known, $u_{2}^{N^{m} n}$ is the control sequence for multi-rate MPC (the decision variables), and $u_{3}^{N^{m} n}$ is a zero vector. Then,

$$
\begin{aligned}
\Phi^{s}(s(k))= & \left(u_{2}^{N^{m} n}\right)^{T} H_{22}^{m} u_{2}^{N^{m} n}(s(k)) \\
& +2\left(u_{2}^{N^{m} n}(s(k))\right)^{T}\left(G_{2}^{m} x(s(k))\right. \\
& \left.+\frac{1}{2}\left(H_{12}^{m T}+H_{21}^{m}\right) u_{1}^{N^{m} n}(s(k))\right)+ \text { constant }
\end{aligned}
$$


Define the objective function for the multi-rate MPC at time $k$ :

$$
\begin{aligned}
\Phi(k)= & \sum_{t=k}^{k+N-1}\left(y_{\text {samp }}(t \mid k)^{T} Q y_{\text {samp }}(t \mid k)\right. \\
& \left.+u_{i}(t \mid k)^{T} R u(t \mid k)\right) \\
& +y_{\text {samp }}(k+N \mid k)^{T} \bar{Q} y_{\text {samp }}(k+N \mid k)
\end{aligned}
$$

where the sampled value of outputs is evaluated instead of their real values obtained from the simulation with the model or process data collected at high frequency, which is the major difference from the regular MPC. Now let $Q=Q^{m} / n, R=R^{m}, \overline{Q_{f}}=\overline{Q_{f}^{m}}$, and also set $u_{3}^{N^{m} n}$ be zero vector as above, then

$$
\Phi(k)=\Phi^{s}(s(k))+\text { constant }
$$

Therefore, since $u^{N} \equiv u_{2}^{N^{m} n}$,

$$
u^{N}(k)=\arg \min _{u^{N}} \Phi(k)=\arg \min _{u_{2}^{N_{n}}} \Phi^{s}(s(k))
$$

Then, Equation 16 converts the control problem of the multi-rate sampled system to the control problem at its sampling time.

\subsection{Multi-rate Distributed Model Predictive Control}

The objective function for subsystem $i$ is defined similarly to Equation 14:

$$
\begin{gathered}
\Phi_{i}(k)=\sum_{t=k}^{k+N-1}\left(y_{\text {samp }, i}(t \mid k)^{T} Q_{i} y_{\text {samp }, i}(t \mid k)+u_{i}(t \mid k)^{T}\right. \\
\left.R_{i} u_{i}(t \mid k)\right)+y_{\text {samp }, i}(k+N \mid k)^{T} \overline{Q_{f, i}} y_{\text {samp }, i}(k+N \mid k)
\end{gathered}
$$

where subscript $i$ denotes the $i t h$ subsystem, and the objective function would be minimized subject to constraints of the system model Equation 7. Use Equation 4 or 8 to generate $H_{i}^{m}$ and $G_{i}^{m}$ in Equation 11, and let the input penalty matrix be $\operatorname{diag}\left(\left[R_{i}, 0_{1 i}\right.\right.$, $\left.\left.\ldots, 0_{(i-1) i}, 0_{(i+1) i}, \ldots, 0_{M i}\right]\right)$, where $0_{j i}$ is the square zeros matrix with the number of rows (or columns) equal to the length of $u_{j i}$.

The overall objective function is the weighted sum of local objective functions:

$$
\Phi=\sum \omega_{i} \Phi_{i}, \quad \text { with } \quad \sum \omega_{i}=1
$$

The centralized solution can be obtained from:

$$
\begin{aligned}
u^{N}(k)= & {\left[u_{1}^{N}(k)^{T}, \cdots, u_{M}^{N}(k)^{T}\right]^{T} } \\
= & \arg \min _{u^{N}} \sum \omega_{i} \Phi_{i}(k) \\
= & \arg \min _{u^{N}} \sum \omega_{i} \Phi^{s}(s(k)) \\
= & \arg \min _{u^{N}}\left(u^{N}(k)^{T} H^{c}(k) u^{N}(k)\right. \\
& \left.+2 u^{N}(k)^{T} G^{c}(k) x(k)\right)
\end{aligned}
$$


The centralized quadratic optimization matrices $H^{c}$ and $G^{c}$ are calculated by summation of corresponding local optimization matrices in Equation 13.

The next step is to decompose the overall objective function back to local objective functions. For subsystem $i$, let $u_{i}^{N}=\Lambda_{i} u^{N}$, then $\Lambda_{i}$ is a matrix with the corresponding

columns of the identity matrix. Define $\overline{u_{i}^{N}}$ to be the inputs of other subsystems, $\overline{\Lambda_{i}}$ to be the other columns of the identity matrix. Assume that the current states and inputs of other subsystems are known. Then, the overall objective function is defined as the new local objective function:

$$
\begin{aligned}
\Phi_{i}^{l}(k)= & u_{i}^{N}(k)^{T} \Lambda_{i} H^{c}(k) \Lambda_{i}^{T} u_{i}^{N}(k)+2 u_{i}^{N}(k)^{T} \\
& {\left[\Lambda_{i} \frac{1}{2}\left(H^{c}(k)+H^{c T}(k)\right) \bar{\Lambda}_{i}^{T} \overline{u_{i}^{N}}(k)+\Lambda_{i} G^{c}(k) x(k)\right] }
\end{aligned}
$$

that can be optimized locally by local controllers, and the input or output constraints can be enforced to the local optimization, which is the same as standard MPC.

With the distributed optimization above, the multi-rate distributed MPC uses the same communication and cooperation structure as the other distributed MPC algorithms in literature such as [3], in which multiple iterations may be conducted in each time step to improve the optimality. The stability in the sense of Lyapunov is guaranteed if $\Phi$ is positive-definite, and

$$
\begin{aligned}
\Phi_{q}(k+1) \leq & \Phi_{0}(k+1) \\
= & \Phi_{q}(k)-\sum_{i=1}^{M}\left(x_{i}^{T}(k) Q_{i} x_{i}(k)\right. \\
& \left.+u_{i, q}^{N^{T}}(k \mid k)(1) R_{i} u_{i, q}^{N}(k \mid k)(1)\right) \\
\leq & \Phi_{q}(k)
\end{aligned}
$$

where $q$ indicates the number of iterations.

\section{Results of Multi-rate Distributed Control}

To demonstrate the effectiveness of the proposed method, two case studies are presented. The first is a linear system artificially sampled at multi-rate, and the second is the control of TE problem based on the identified model obtained in previous sections.

\subsection{Distillation Column}

For a system with two subsystems described by basic- rate system matrices, each subsystem has one input and one output. This is a distillation column model from [3], with two manipulated variables (vapor boilup flowrate and reflux flowrate, respectively) and two controlled variables (temperatures of tray 27 and 7 ).

Now arbitrarily assign different sampling times to controlled variables, and construct the lifted model for each subsystem by using Equation 6 . Then pretend that only the 
lifted models are known, and try to control this system by distributed control. The control objective is to make target tracking with a new setpoint $y_{t}=[1,-1]^{T}$, under the hard constraints for inputs $-1.5 \leq u_{1} \leq 1.5$ and $-2 \leq u_{2} \leq 2$.

Before examining the performance of multi-rate distributed control, the multi-rate centralized control is studied to evaluate MPC by lifted model. Make a centralized model for centralized control:

$$
A_{c}=\left[\begin{array}{cc}
A_{1} & 0 \\
0 & A_{2}
\end{array}\right] \quad B_{c}=\left[\begin{array}{cc}
B_{1} & W_{12} \\
W_{21} & B_{2}
\end{array}\right] \quad C_{c}=\left[\begin{array}{cc}
C_{1} & 0 \\
0 & C_{2}
\end{array}\right]
$$

Set the sampling time to $n_{c}=11$, and construct the lifted model for the centralized control system, using model predictive control with quadratic objective function. The quadratic optimizations were done by quadprog in Matlab. The results are shown in Fig. 5(a), in which the basic-rate response (dashed line) has many fluctuations because the lifted model has no information about the intermediate status other than at sampling points. The solid line shows the sampled value, and a smooth response is obtained as expected. For the input side, the periodical saw-tooth shape has the same period as sampling, and it is an aggressive behavior for this system with fast dynamics.

The fluctuations for both outputs and inputs are observed for control with lifted model, however it may not be so severe in systems with slower dynamics. In case such fluctuations are not desirable, zero-order hold $(\mathrm{ZOH})$ can be added as the constraints for the inputs, and the stability is still ensured since Equation 21 still holds. The $\mathrm{ZOH}$ for inputs can be realized by adding the following equality constraints:

$$
\left[\begin{array}{lll}
Z & & 0 \\
& \ddots & \\
0 & & Z
\end{array}\right] u^{N}=\left[\begin{array}{c}
0 \\
\vdots \\
0
\end{array}\right]
$$

The number of $Z$ in the diagonal of the left matrix is $N^{m}$, and

$$
Z=\left[\begin{array}{ccccc}
1 & -1 & 0 & \ldots & 0 \\
1 & 0 & -1 & \ldots & 0 \\
\vdots & \vdots & \vdots & \ddots & \vdots \\
1 & 0 & 0 & \ldots & -1
\end{array}\right]
$$

and the dimension of $Z$ is $(n-1) \times n$, where $n$ is the sampling steps. The control results for centralized control with $\mathrm{ZOH}$ is shown in Fig. 5(b), in which smooth response is obtained.

Next we pretend that the system model is unknown, and subspace identification is conducted for the multi-rate sampling system to get the lifted model. For simplicity, Gaussian inputs are used to excite the process, and white noise is added to the system artificially. After the lifted model is identified, the states at a sampling time can be estimated by a Kalman filter (fixed gain Kalman filter is used in this simulation for simplicity), and then the control system calculates the control actions by using the estimated states. 

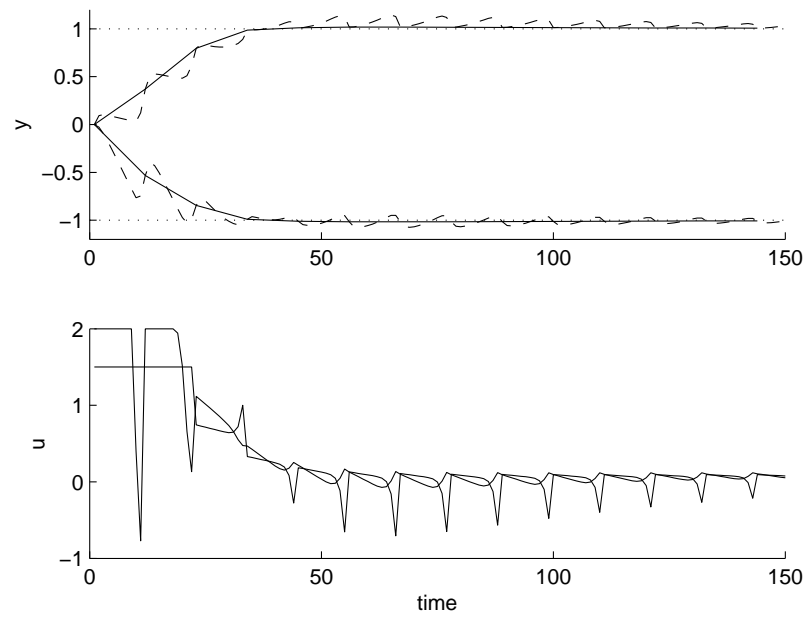

(a) Without zero-order hold.
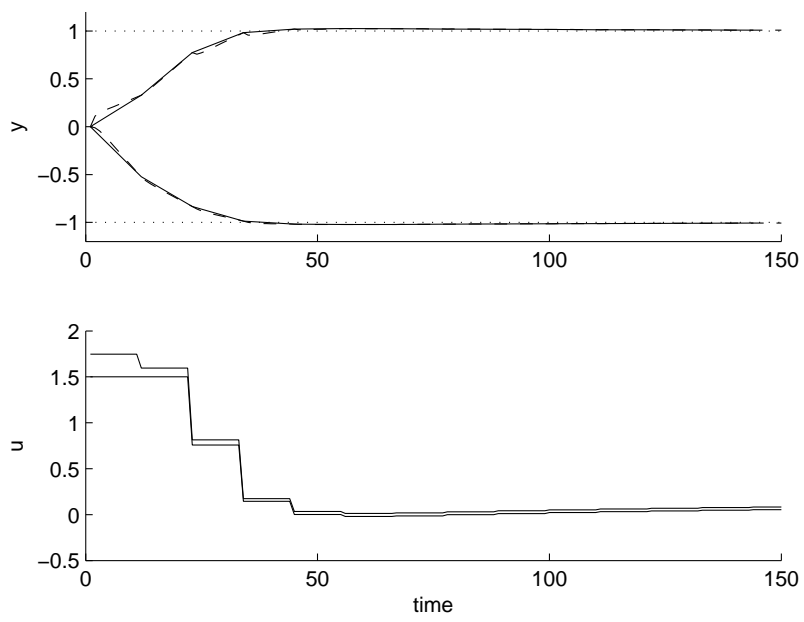

(b) With zero-order hold.

Figure 5: Results of centralized multi-rate control. For outputs, the dashed line shows basic-rate response, and the solid line shows the measurements at sampling times. 
The identified model for different noise levels in identification is applied to the system without noise, to test the quality of each model. Noise level is defined as a noise to signal ratio $($ noise/signal $\times 100 \%)$. The effects of noise levels for four cases $(0.2 \%, 1 \%, 5 \%$, and $25 \%$ noise level) are illustrated in Figure 6. For small noises, the identified model achieved identical control results as the exact model, and as the noise increases, the responses degrade both in terms of dynamics (slower response) and steady state offset.
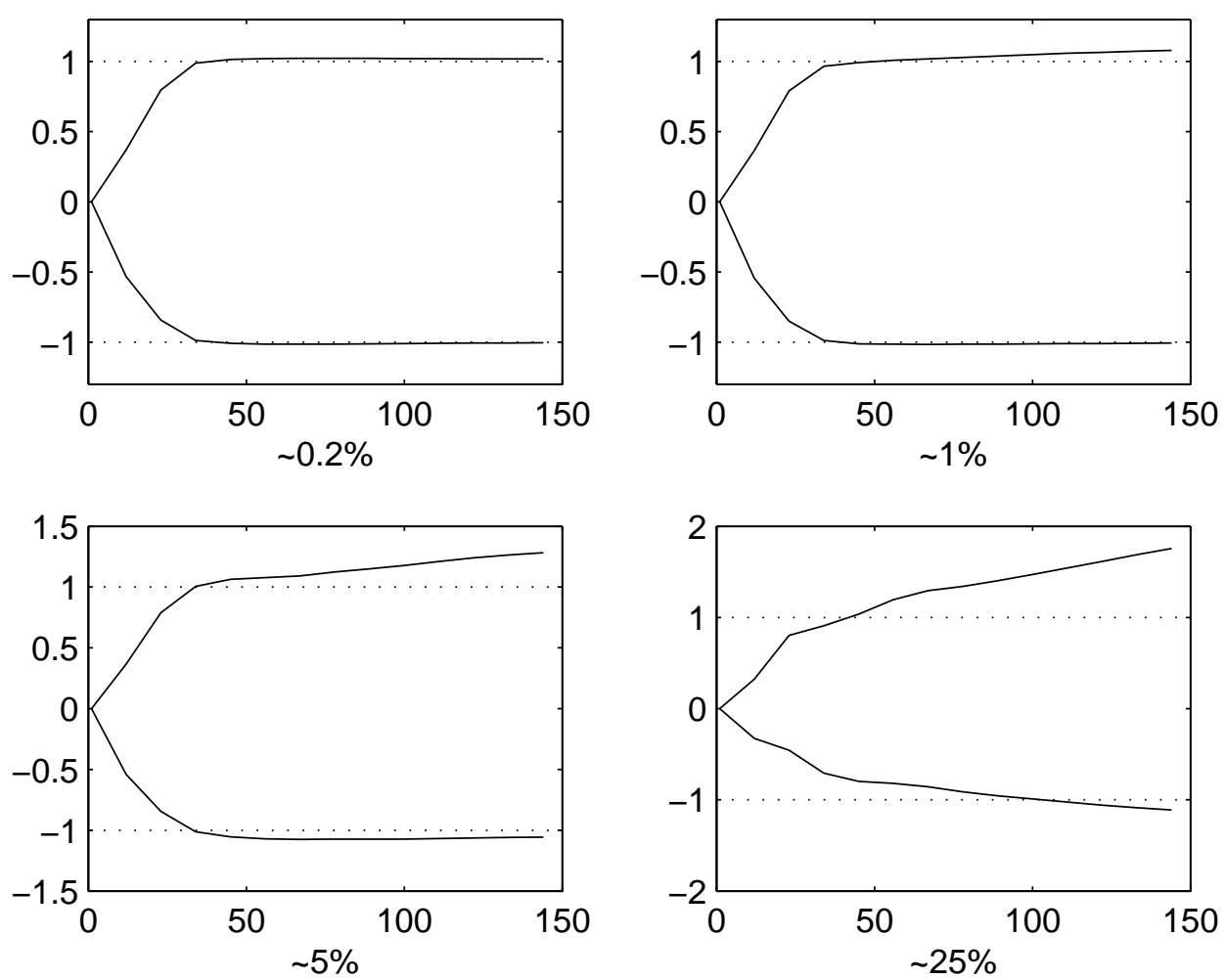

Figure 6: The control results of identified models of different noise level.

Finally, distributed control approach is illustrated. Sampling time of these two subsystems are set to $n_{1}=3$ and $n_{2}=7$. Take 10 iterations (maximum $q$ equals to 10 ) at each sampling time, the results with and without $\mathrm{ZOH}$ are shown in Fig. 7, similar but worse responses are obtained in both cases compared to the centralized control, and even the $\mathrm{ZOH}$ constrained case shows some fluctuations. These observations are mainly because of the different sampling times of the two subsystems, but not distributed control, since 10 iterations could generate results that are close enough to the centralized control, from the study of basic-rate distributed control on this system.

Notice that the formulation of constraints is different from the centralized control case. Denote the equality matrix in Equation 23 as $\Xi$, and make the same partition as Equation 

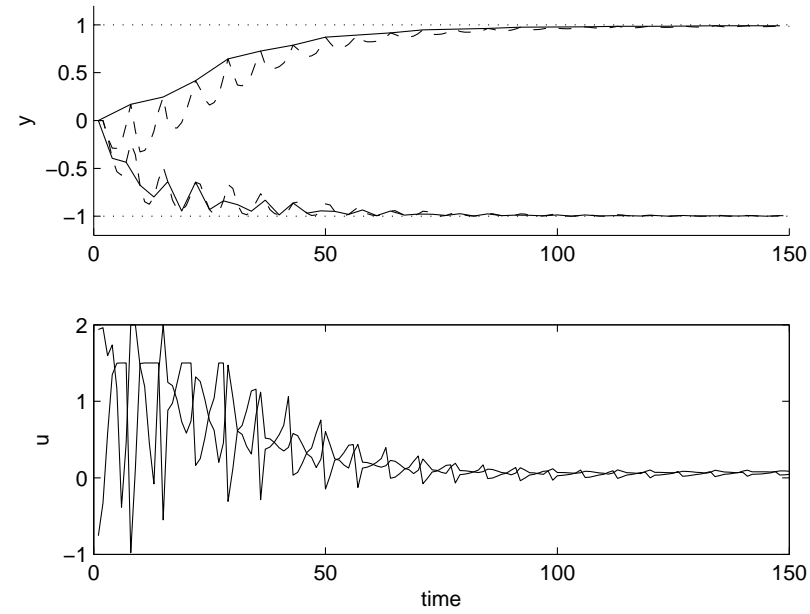

(a) Without zero-order hold.
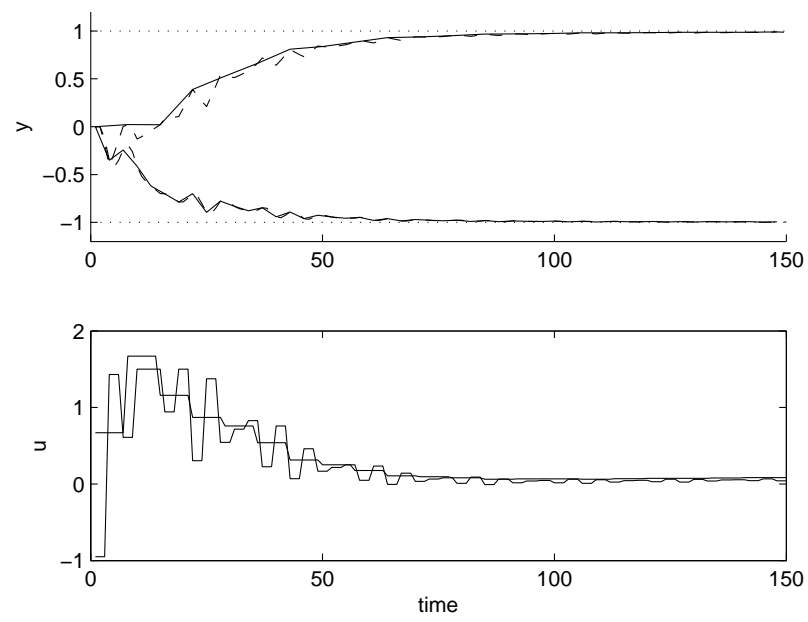

(b) With zero-order hold.

Figure 7: Results of distributed multi-rate control. For outputs, the dashed line shows basic-rate response, and the solid line shows the sampling measurements. 
12,

$$
\Xi=\left[\begin{array}{lll}
\Xi_{11} & \Xi_{12} & \Xi_{13} \\
\Xi_{21} & \Xi_{22} & \Xi_{23} \\
\Xi_{31} & \Xi_{32} & \Xi_{33}
\end{array}\right]
$$

then the constraints become:

$$
\Xi_{22} u_{2}^{N^{m} n}=-\Xi_{11} u_{1}^{N^{m} n}
$$

and $u_{2}^{N^{m} n}$ is actually the decision variable in the optimization described in Equation 16, while $u_{1}^{N^{m} n}$ is known.

A comparison of distributed multi-rate control with $\mathrm{ZOH}$, without $\mathrm{ZOH}$, and centralized basic-rate control is given in Fig. 8, in which only sampled values are plotted. The centralized basic-rate control has the best control results, and control without constraints generates slightly better response compared to the constrained case as expected, at the expense of more fluctuations.

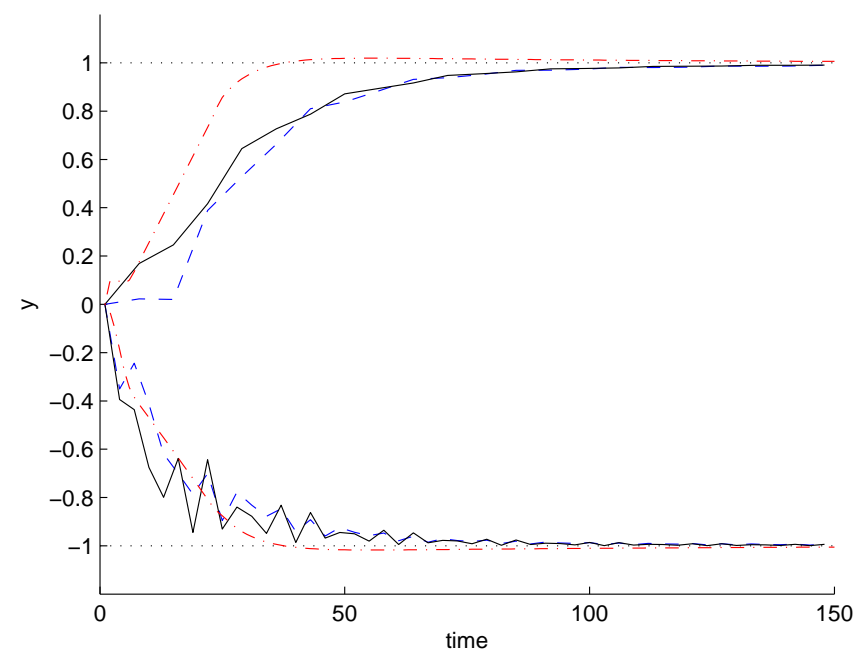

Figure 8: Comparison of different control method. The solid black line is the response of distributed multirate control without zero-order hold, the dashed blue line is the response of distributed multi-rate control with zero-order hold, and the dash-dotted red line is the response of centralized control for basic rate.

\subsection{Tennessee Eastman Challenge Problem}

In this section, the proposed multi-rate distributed control is implemented on TE process based on the identified model. Because of the poor predictions of the overall model (especially for the G concentration, see Figure 2), the control based on the overall model cannot achieve good results. An example is shown in Figure 9, which uses similar setting as distributed control case that will be discussed later, but fails to get reasonable tracking results compared to Figure 11(a). 

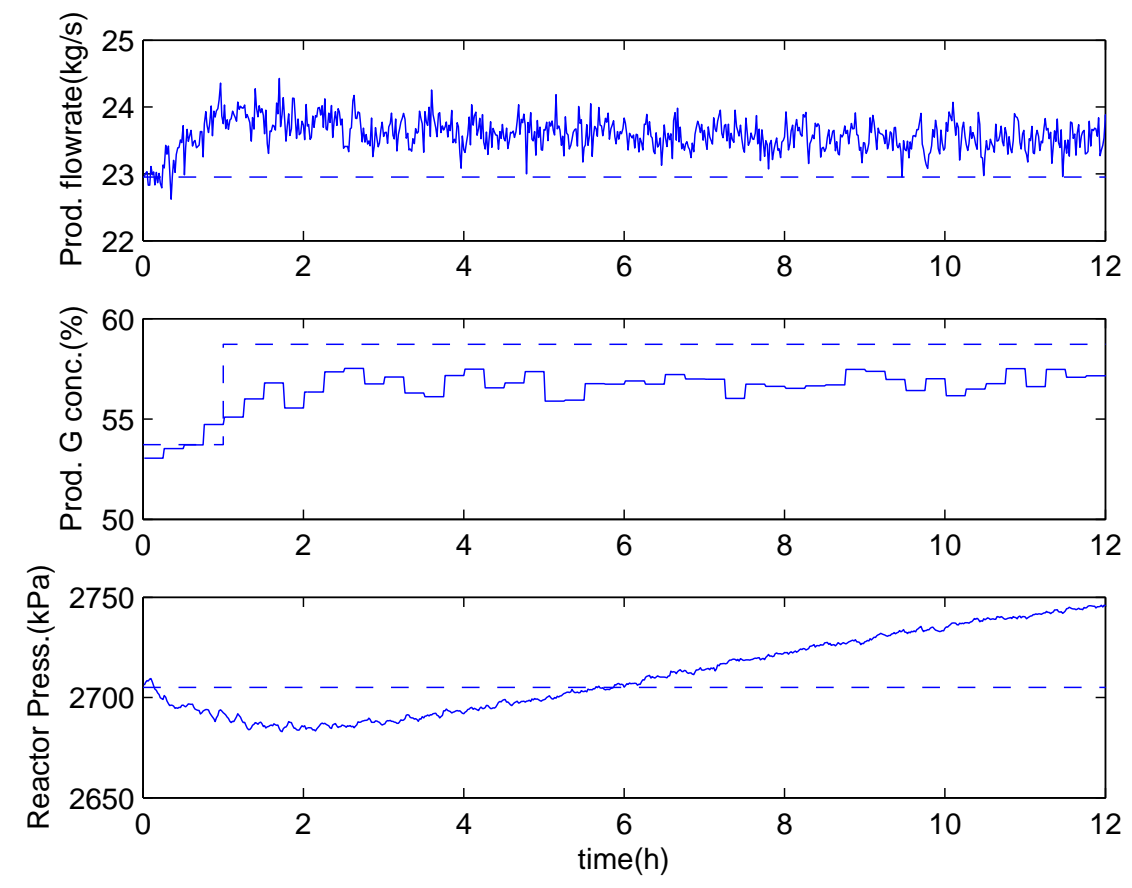

Figure 9: Overall control for target tracking, a step setpoint change of $+5 \%$ in $\mathrm{G}$ concentration in product.

From the previous study, it is obvious that the reactor pressure response is nonlinear to all the MVs, and to reduce such nonlinearity, offset-free MPC [39, 40, 41, 42] technique is implemented on the reactor pressure. Only consider output offset in disturbance model in offset-free MPC, and consider the inputs from neighbor subsystems. The estimated states could be obtained from the Kalman filter. Then the output offset is

$$
p(k)=y(k)-C \hat{x}(k)
$$

where $\hat{x}$ denotes estimated states from Kalman filter. However, the above estimation of offset may be too aggressive, and one can apply exponential moving average on $p(k)$ :

$$
\hat{p}(k)=(1-\gamma) \hat{p}(k-1)+\gamma p(k)
$$

where $\gamma$ is the forgetting factor between 0 and 1, which is used to smooth out the estimate of the offset. Then, the new output target at each step would be $y_{t}-\hat{p}(k)$.

\subsubsection{Setpoint tracking near operating point}

The first case makes relatively small changes near original setpoints to check the performance of DMPC controller. Specifically, setpoint step change of $+100 \mathrm{kPa}$ in reactor pressure, step change of $+5 \%$ in G concentration in product, and step change of $-1 \mathrm{~m}^{3} / h$ in product flowrate are tested separately.

Reactor pressure tracking. The control objective is to track the setpoint step change of $+100 \mathrm{kPa}$ in the reactor pressure, which is the simplest case since the pressure is very 
sensitive to all the controlled variables, and it works well even without offset-free control. The results are shown in Figure 10, in which the dashed line is the setpoint, and solid line is the response of the process. Hard constraints is applied to the MVs, and the figure indicates that the purge flowrate has reached its lower limit when the setpoint was just changed. Also, the figure shows that all the setpoints could be tracked well.

Product $\mathrm{G}$ concentration tracking. The control objective is to track the setpoint step change of $+5 \%$ in $\mathrm{G}$ concentration in product. The results without offset-free control and with offset-free control are shown in Figure 11, and manipulated variables are not shown for conciseness. Without offset-free control, the pressure appears an offset of about $10 \mathrm{kPa}$; while the $\mathrm{G}$ concentration also has some minor offset, which is not significant, but it is also caused by the offset of pressure. With offset-free control, the pressure is kept around the setpoint, and so is the $\mathrm{G}$ concentration. The dynamics of the response improve because the offset-free control was trying to compensate the offset.

Product flowrate tracking. The control objective of the last scheme in this subsection is to track the setpoint step change of $-1 \mathrm{~m}^{3} / h$ in product flowrate. The study of the CVs' responses to MVs show that the product flowrate is difficult to be controlled by current MVs, and just $-1 \mathrm{~m}^{3} / \mathrm{h}$ is a relative large change for it. The control results are shown in Figure 12, for which offset-free controls for both reactor pressure and G concentration are applied, however minor offset for reactor is still observable. The difficulty of product flowrate control brings trouble when large concentration changes are made, thus it is dropped from the CVs for target tracking, but still a CV in the MPC control. In [29] similar treatment could be found.

\subsubsection{Setpoints tracking for large setpoint changes and mode change}

In TE process, the most important controlled variable is the concentration of product G. Since it has a large sampling time, it is difficult to predict by the identified model from traditional subspace identification technique. The method proposed in this work successfully solves the multi-rate sampling problem, and large setpoint changes are studied in this subsection.

Product $\mathrm{G}$ concentration to $75 \%$ and $35 \%$. These setpoint changes here are large but not extreme, and as discussed previously, the product flowrate is not considered when calculating the new setpoints for target tracking. For both cases, offset-free control is applied on the reactor pressure, and the system response could successfully follow the changes of setpoints without offset (Figure 13).

Change to mode 90/10. The new setpoint for product $\mathrm{G}$ concentration is $90.09 \%$ in this case. In this extreme case, two problems arise when applying offset-free control on reactor pressure:

1. If the forgetting factor $\gamma$ is small, the dynamics of the offset-free policy will be slow and would yield large variation from the setpoints.

2. If $\gamma$ is large, then the prediction error $\hat{p}(k)$ will be fluctuating around the average value in this case. This situation is shown in Figure 14(a). 

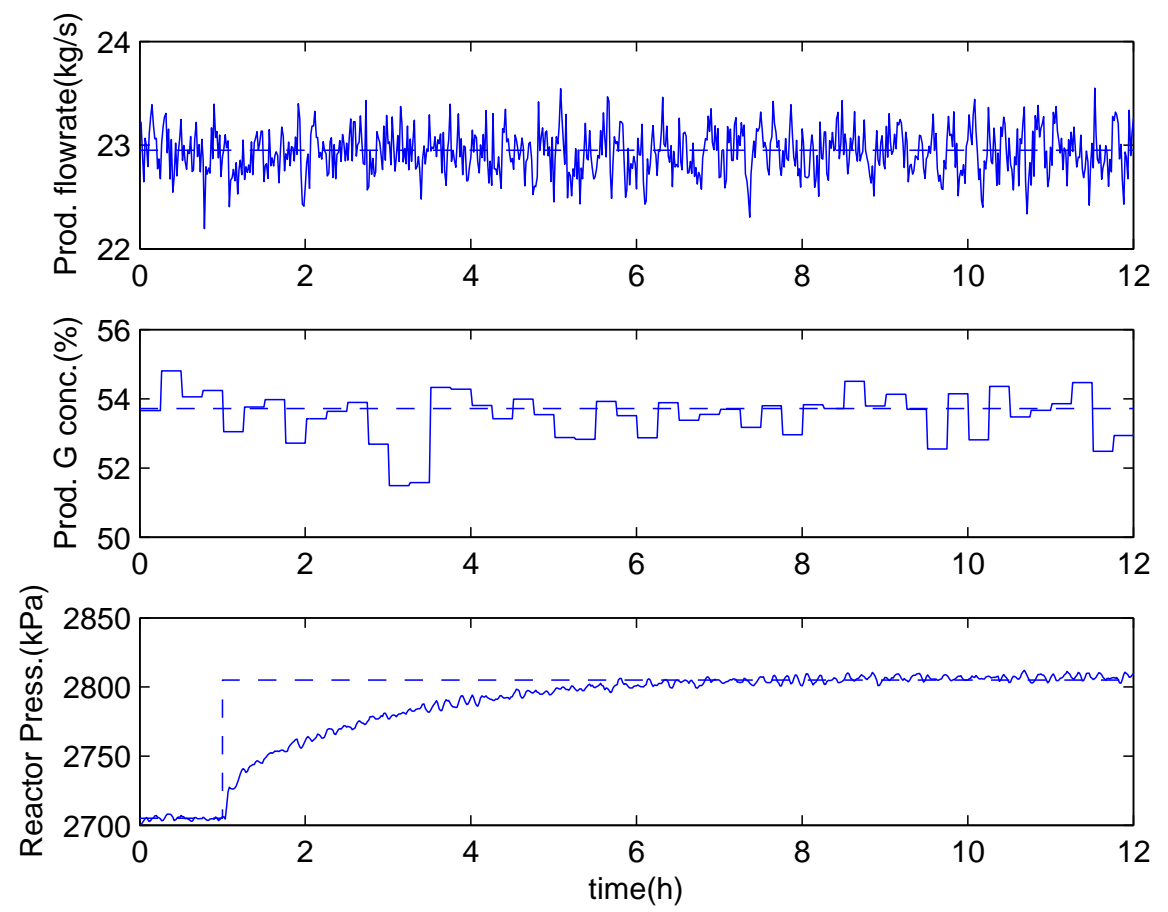

(a) Controlled variables.

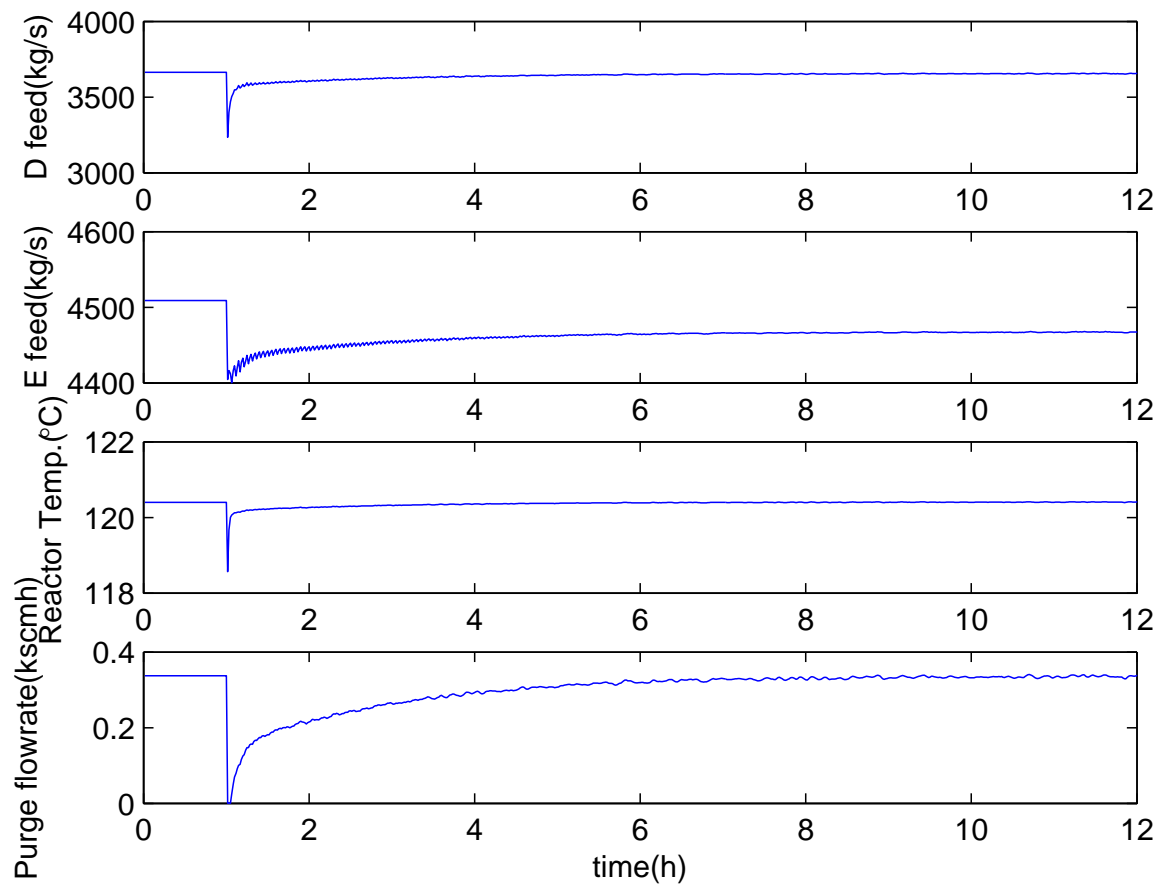

(b) Manipulated variables.

Figure 10: Distributed control for target tracking, a step setpoint change of $+100 \mathrm{kPa}$ in reactor pressure. 

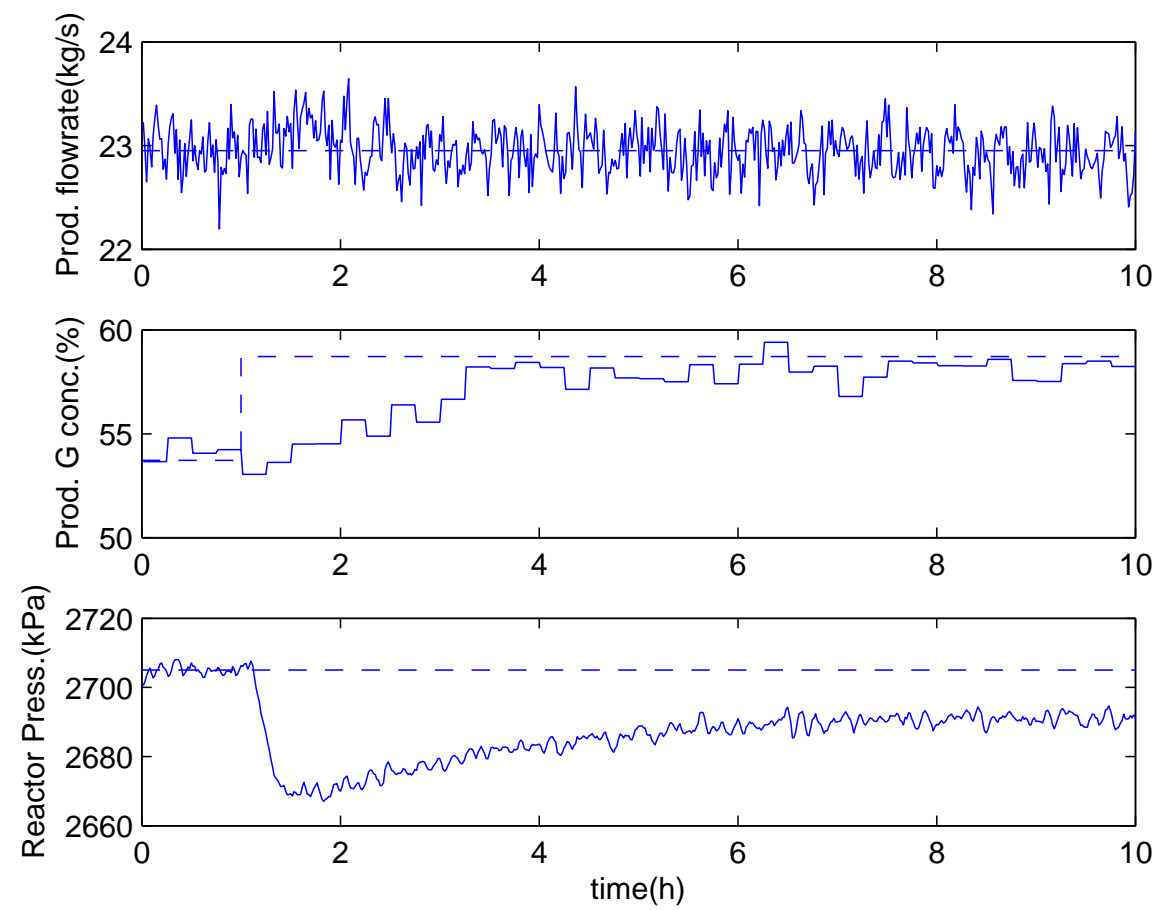

(a) Without offset-free control.
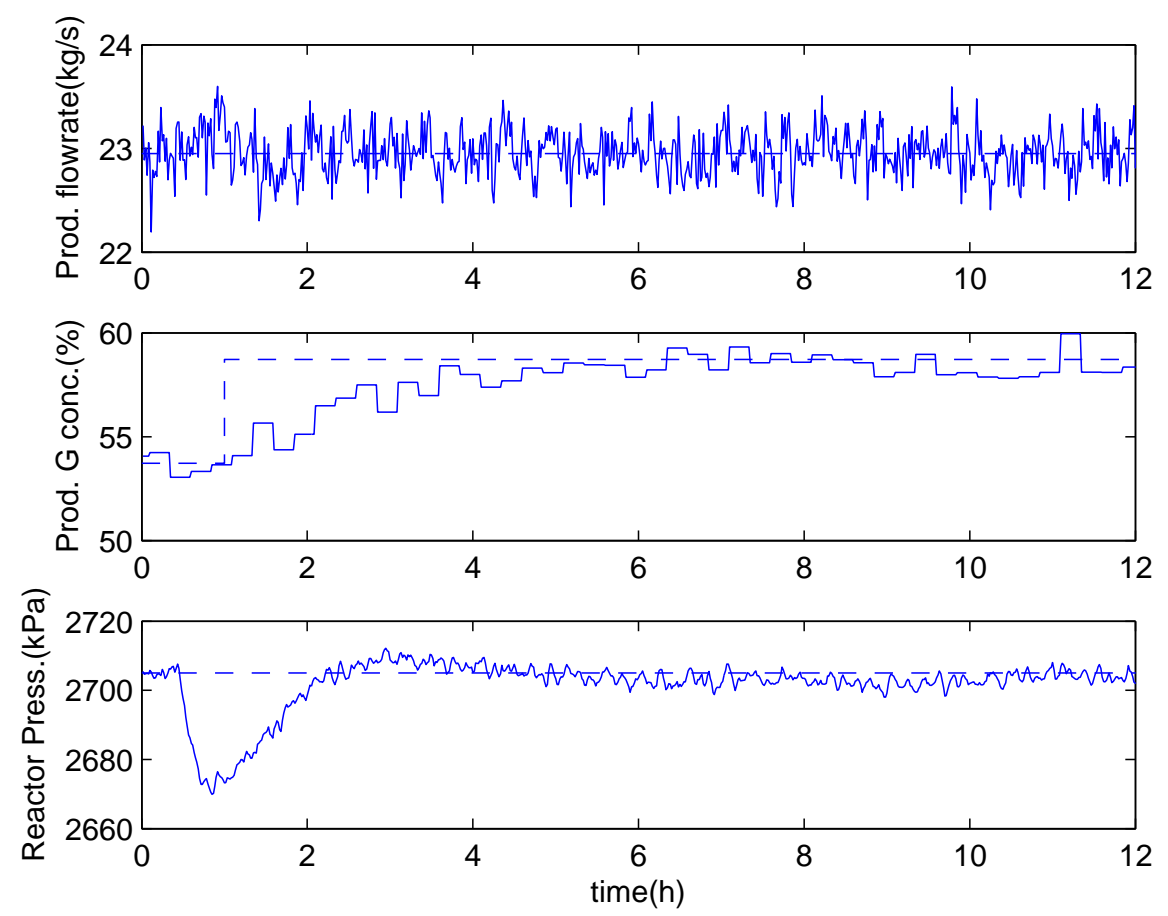

(b) With offset-free control.

Figure 11: Distributed control for target tracking, a step setpoint change of $+5 \%$ in $\mathrm{G}$ concentration in product. 

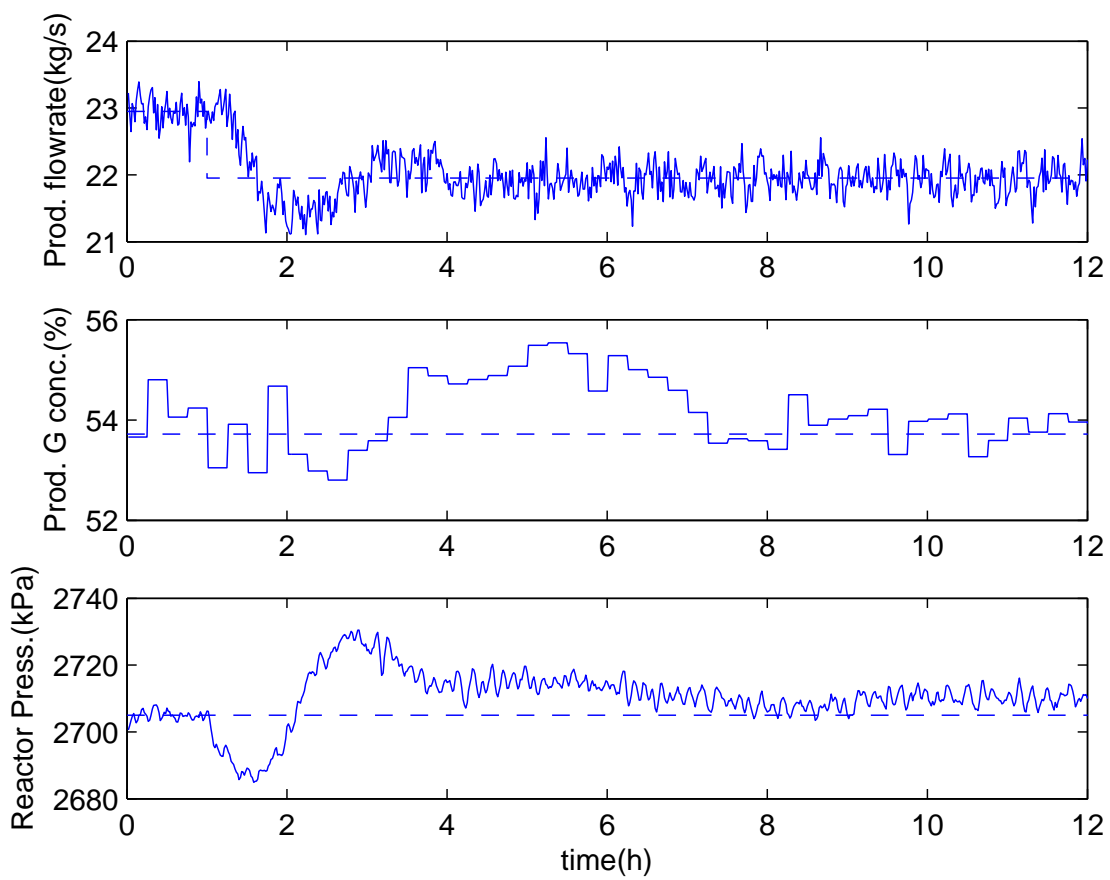

Figure 12: Distributed control for target tracking with offset-free control, a step setpoint change of $-1 \mathrm{~m}^{3} / \mathrm{h}$ in product flowrate.

The solution is to use adaptive $\gamma$. When the setpoint change is made, first use large $\gamma$ for fast response, and then change to small $\gamma$ when the system response is converging. The controller is calculating both the offset value during the start period of the setpoint change, and determining automatically the sign of the offset after a small period of time (in this case is negative). The criterion for $\gamma$ switch is:

$$
\gamma_{E W M A} \times \operatorname{sign}<0
$$

The results are shown in Figure 14(b), where some fluctuations remain, however the response matches the observation in Figure 14(a) for $\gamma=1 / 60$. This is because a moderate value of $\gamma$ is used, and further decrease of $\gamma$ could reduce the fluctuation further.

Change to mode 10/90. The new setpoint for product $\mathrm{G}$ concentration is $11.88 \%$, and the results are shown in Figure 15. The setpoints could not be reached, because the maximum ability of the MVs has been reached: the $\mathrm{E}$ feed has reached its maximum, and further reduce $\mathrm{G}$ concentration requires further decrease of $\mathrm{D}$ feed, however, reducing $\mathrm{D}$ feed will cause the reactor pressure increases, but the reactor temperature and purge flowrate have reached their upper limits to bring the reactor pressure down.

\section{Conclusions}

A system identification and distributed control method is proposed in order to handle the problems caused by conducting subspace system identification on multi- rate sampled 

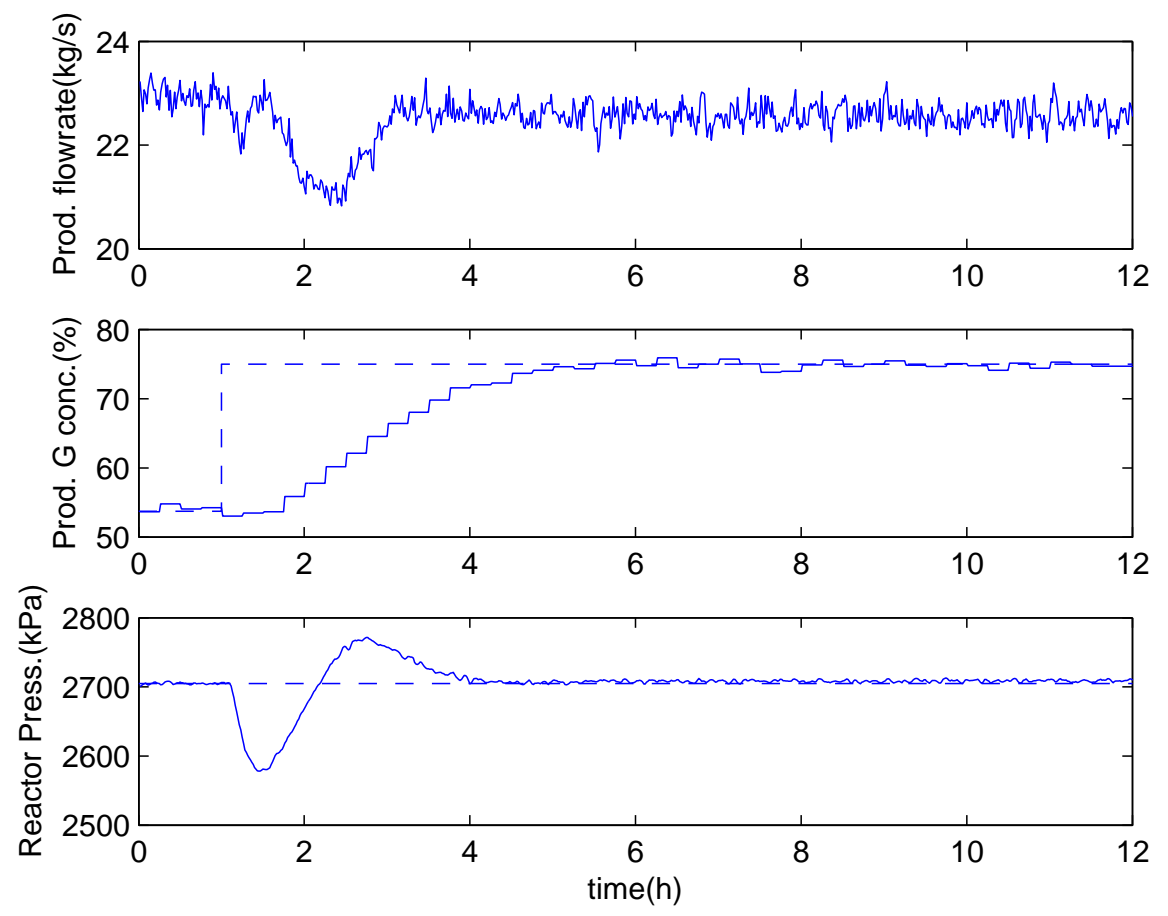

(a) Product G concentration to $75 \%$.
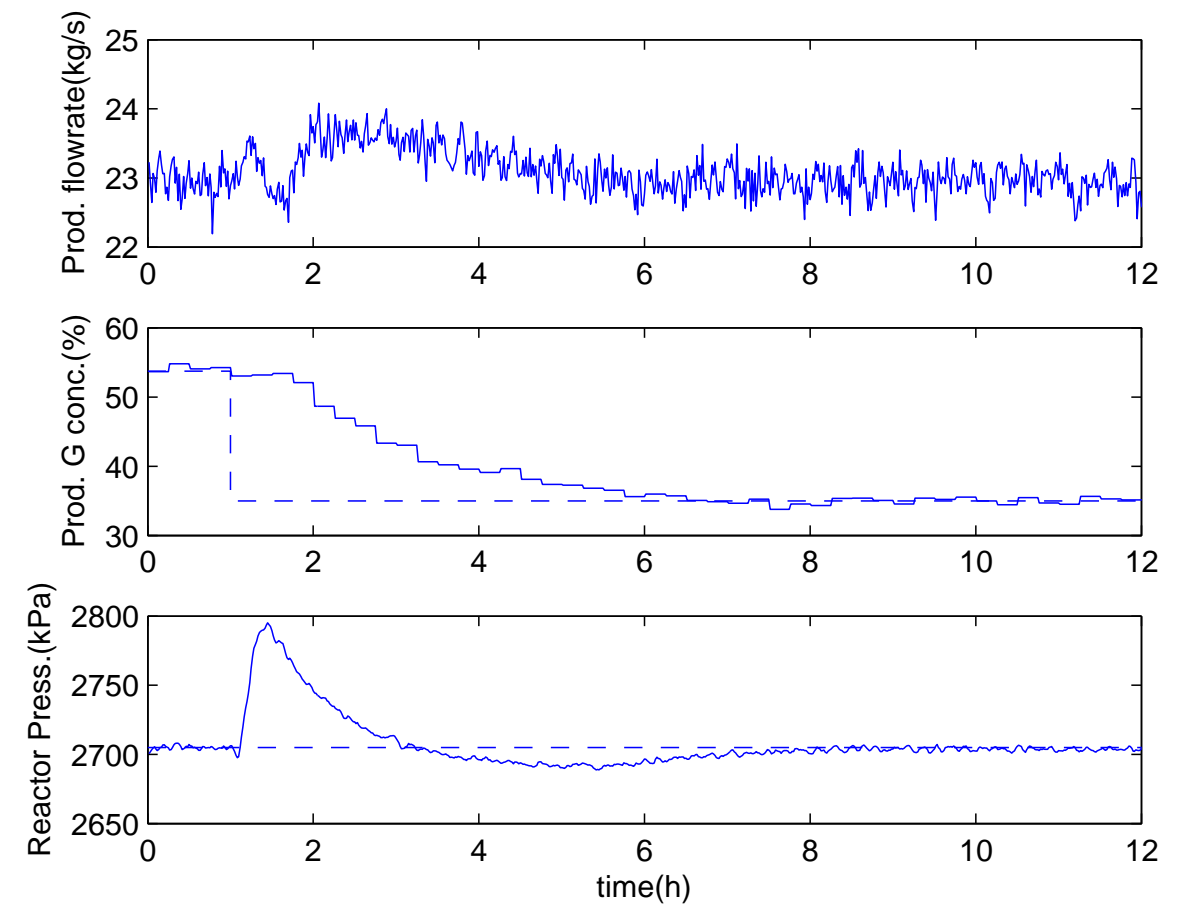

(b) Product G concentration to $35 \%$.

Figure 13: Distributed control for target tracking, product G concentration is changed to $75 \%$ and $35 \%$. 


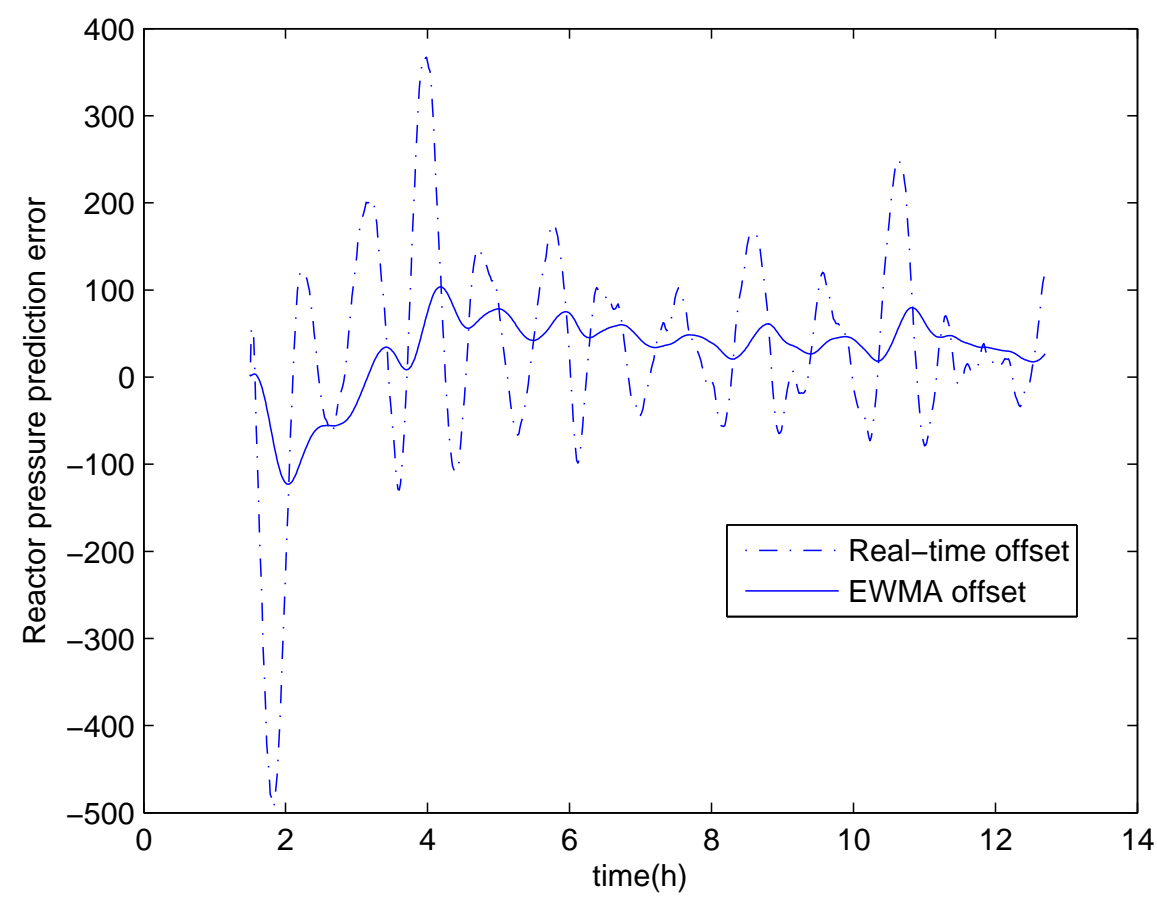

(a) The pressure offset in the case to mode 90/10. The dot-dashed line is the real-time offset, i.e. $\gamma=1$, and the solid line shows the EWMA offset when $\gamma=1 / 60$.
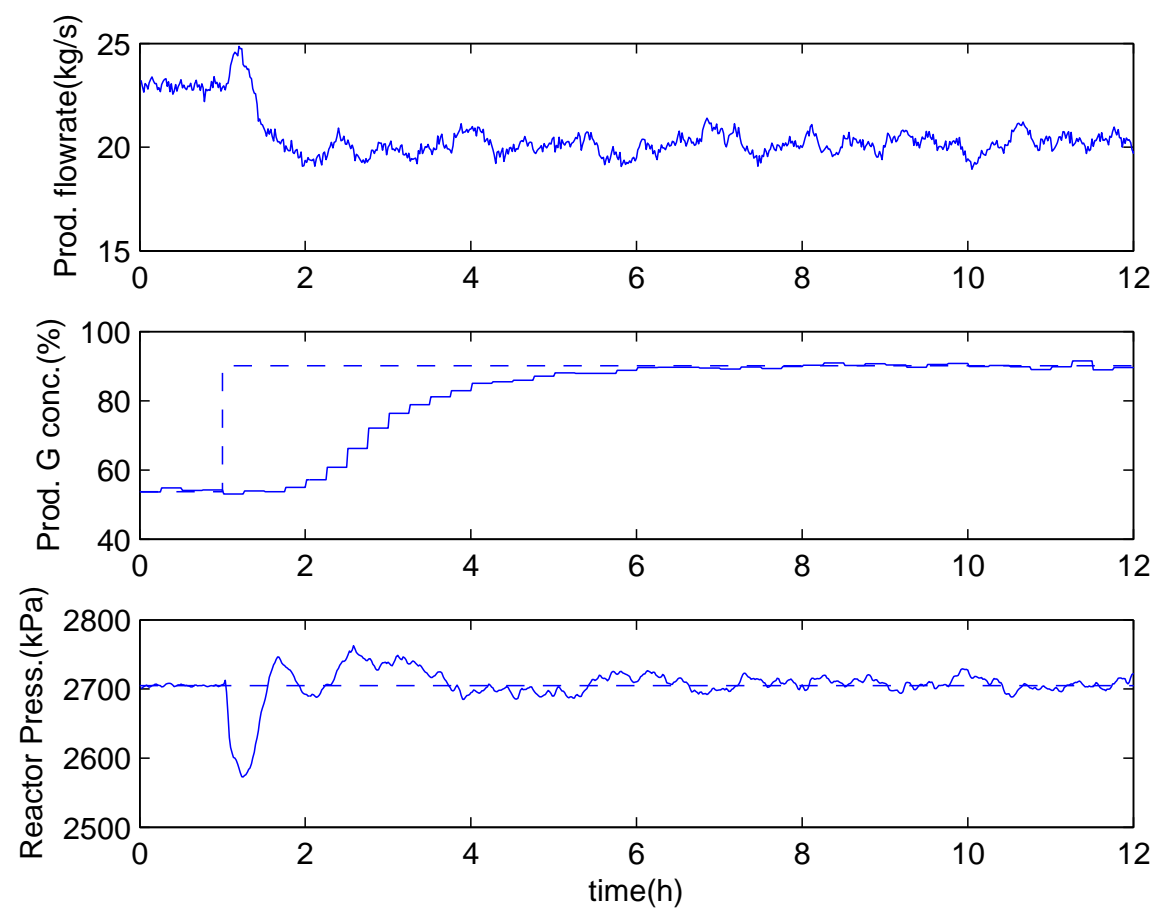

(b) To mode $90 / 10$ with adaptive $\gamma$.

Figure 14: Distributed control for mode change to 90/10. 

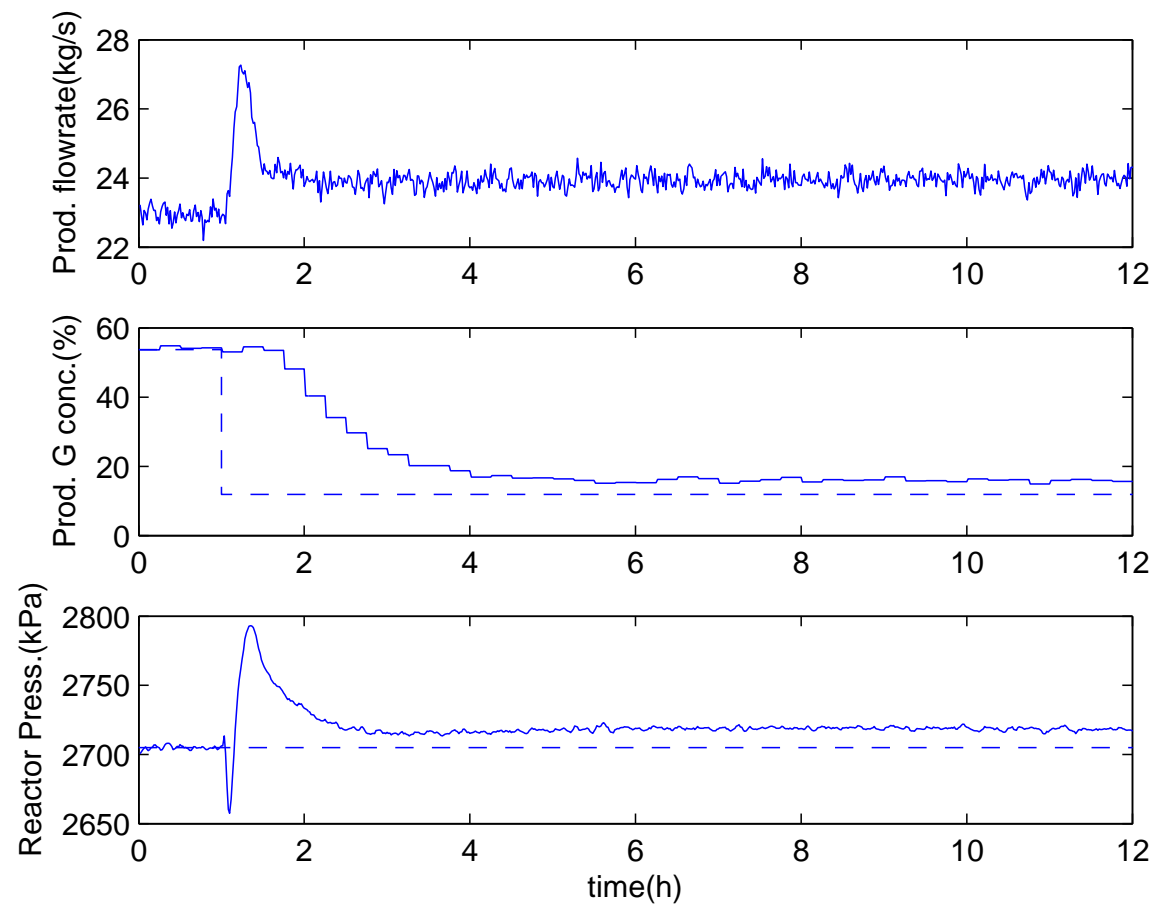

(a) Controlled variables.
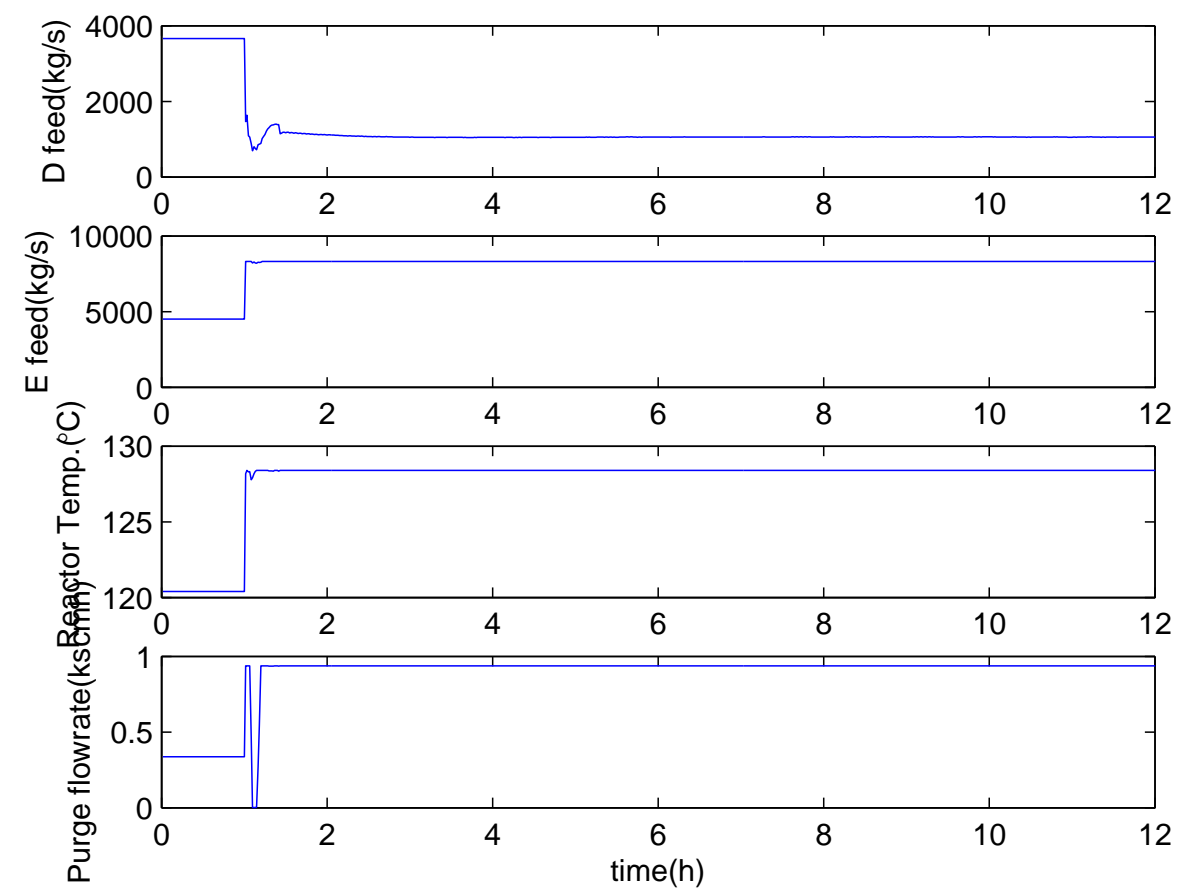

(b) Manipulated variables.

Figure 15: Distributed control for mode change to 10/90. 
systems. The proposed method is illustrated by applying to an artificial multi-rate linear system and the TE challenge problem. The results indicate that the proposed method can yield better models and improve the control of multi-rate sampled systems by using the identified model. The multi-rate modeling, coordination of local controllers and distributed control activities can be carried out with an agent-based system such as MADCABS.

Acknowledgments - Partial support of the research conducted by National Science Foundation Grant CTS-0325378 of the ITR program is gratefully acknowledged.

\section{References}

[1] P. Qin, Z.-J. Yang, R. Nishii, Predictive control for dual-rate systems based on lifted state-space model identified by n4sid method, in: Decision and Control, 2009 held jointly with the 2009 28th Chinese Control Conference. CDC/CCC 2009. Proceedings of the 48th IEEE Conference on, 2009, pp. 73697374.

[2] D. Li, Identification of fast-rate models from multirate data, Int. J. Control 74 (2001) 680-689.

[3] A. N. Venkat, J. B. Rawlings, S. J. Wright, Stability and optimality of distributed, linear model predictive control part i: state feedback, Tech. rep., University of Wisconsin, Madison (2006).

[4] A. N. Venkat, J. B. Rawlings, S. J. Wright, Stability and optimality of distributed, linear model predictive control part ii: Output feedback, Tech. rep., University of Wisconsin, Madison (2006).

[5] A. N. Venkat, J. B. Rawlings, S. J. Wright, Stability and optimality of distributed model predictive control, in: Decision and Control, 2005 and 2005 European Control Conference. CDC-ECC '05. 44th IEEE Conference on, 2005, pp. 6680-6685.

[6] B. T. Stewart, A. N. Venkat, J. B. Rawlings, S. J. Wright, G. Pannocchia, Cooperative distributed model predictive control, Systems \& Control Letters 59 (8) (2010) 460 - 469.

[7] J. Liu, D. Muñoz de la Peña, P. D. Christofides, Distributed model predictive control of nonlinear process systems, AIChE Journal 55 (5) (2009) 1171-1184.

[8] P. D. Christofides, R. Scattolini, D. Muñoz de la Peña, J. Liu, Distributed model predictive control: A tutorial review and future research directions, Computers \& Chemical Engineering 51 (2013) 21-41.

[9] J. B. Rawlings, B. T. Stewart, Coordinating multiple optimization-based controllers: New opportunities and challenges, Journal of Process Control 18 (9) (2008) 839 - 845.

[10] B. T. Stewart, S. J. Wright, J. B. Rawlings, Cooperative distributed model predictive control for nonlinear systems, Journal of Process Control 21 (5) (2011) 698 - 704, special Issue on Hierarchical and Distributed Model Predictive Control.

[11] J. Liu, X. Chen, D. Muñoz de la Peña, P. D. Christofides, Sequential and iterative architectures for distributed model predictive control of nonlinear process systems, AIChE Journal 56 (8) (2010) 21372149.

[12] P. D. Christofides, J. Liu, D. M. De La Pena, Networked and distributed predictive control: Methods and nonlinear process network applications, Springer, 2011.

[13] M. Heidarinejad, J. Liu, D. M. noz de la Peña, J. F. Davis, P. D. Christofides, Multirate lyapunov-based distributed model predictive control of nonlinear uncertain systems, Journal of Process Control 21 (9) (2011) $1231-1242$.

[14] Y. Sun, N. H. El-Farra, Quasi-decentralized model-based networked control of process systems, Computers \& Chemical Engineering 32 (9) (2008) 2016 - 2029, networked and Complex Systems S.I. Control of Networked and Complex Process Systems.

[15] E. Camponogara, D. Jia, B. H. Krogh, S. Talukdar, Distributed model predictive control, Control Systems, IEEE 22 (1) (2002) 44-52.

[16] L. Magni, R. Scattolini, Stabilizing decentralized model predictive control of nonlinear systems, Automatica 42 (7) (2006) 1231-1236. 
[17] A. Alessio, D. Barcelli, A. Bemporad, Decentralized model predictive control of dynamically coupled linear systems, Journal of Process Control 21 (5) (2011) 705 - 714, special Issue on Hierarchical and Distributed Model Predictive Control.

[18] M. D. Doan, T. Keviczky, B. D. Schutter, An iterative scheme for distributed model predictive control using fenchel's duality, Journal of Process Control 21 (5) (2011) 746 - 755, special Issue on Hierarchical and Distributed Model Predictive Control.

[19] A. Ferramosca, D. Limon, I. Alvarado, E. Camacho, Cooperative distributed $\{\mathrm{MPC}\}$ for tracking, Automatica 49 (4) (2013) 906 - 914.

[20] R. Scattolini, Architectures for distributed and hierarchical model predictive control c a review, Journal of Process Control 19 (5) (2009) $723-731$.

[21] R. Scattolini, N. Schiavoni, A multirate model-based predictive controller, Automatic Control, IEEE Transactions on 40 (6) (1995) 1093-1097.

[22] S. Roshany-Yamchi, R. R. Negenborn, M. Cychowski, B. De Schutter, J. Connell, K. Delaney, Distributed model predictive control and estimation of large-scale multi-rate systems, in: Proceedings of the 18th IFAC World Congress, 2011.

[23] S. Roshany-Yamchi, M. Cychowski, R. R. Negenborn, B. De Schutter, K. Delaney, J. Connell, Kalman filter-based distributed predictive control of large-scale multi-rate systems: Application to power networks, Control Systems Technology, IEEE Transactions on 21 (1) (2013) 27-39.

[24] J. J. Downs, E. F. Vogel, A plant-wide industrial process control problem, Computers \& Chemical Engineering 17 (3) (1993) 245-255.

[25] N. L. Ricker, Optimal steady-state operation of the tennessee eastman challenge process, Computers \& Chemical Engineering 19 (9) (1995) 949-959.

[26] N. L. Ricker, J. H. Lee, Nonlinear modeling and state estimation for the tennessee eastman challenge process, Computers \& Chemical Engineering 19 (9) (1995) 983-1005.

[27] N. L. Ricker, J. H. Lee, Nonlinear model predictive control of the tennessee eastman challenge process, Computers \& Chemical Engineering 19 (9) (1995) 961-981.

[28] N. L. Ricker, Decentralized control of the tennessee eastman challenge process, Journal of Process Control 6 (4) (1996) 205-221.

[29] R. G. Sriniwas, Y. Arkun, Control of the tennessee eastman process using input-output models, Journal of Process Control 7 (5) (1997) 387-400.

[30] B. C. Juricek, D. E. Seborg, W. E. Larimore, Identification of the tennessee eastman challenge process with subspace methods, Control Engineering Practice 9 (12) (2001) 1337-1351.

[31] E. Tatara, F. Teymour, A. Cinar, Control of complex distributed systems with distributed intelligent agents, J Process Control 17 (5) (2007) 415-427.

[32] S. Perk, F. Teymour, A. Cinar, Statistical monitoring of complex chemical processes using agent-based systems, Industrial and Engineering Chemistry Research 49 (2011) 5080-5093.

[33] A. Artel, F. Teymour, M. North, A. Cinar, A multi-agent approach using perceptron-based learning for robust operation of distributed chemical reactors, Engng Applications of Artificial Intelligence 24 (6) (2011) 1035-1045.

[34] S. Perk, F. Teymour, A. Cinar, An adaptive agent-based system for process fault classification and diagnosis, Industrial and Engineering Chemistry Research 50 (15) (2011) 9138-9155.

[35] S. Perk, Q. M. Shao, F. Teymour, A. Cinar, An adaptive fault-tolerant control framework with agentbased systems, International Journal of Robust and Nonlinear Control 22 (2012) 43-67.

[36] Q. M. Shao, A. Cinar, Coordination scheme and target tracking for distributed model predictive control, accepted by Chemical Engineering Science (2015).

[37] W. E. Larimore, Statistical optimality and canonical variate analysis system identification, Signal Processing 52 (2) (1996) 131-144.

[38] S. J. Qin, An overview of subspace identification, Computers \& Chemical Engineering 30 (10-12) (2006) $1502-1513$.

[39] K. R. Muske, J. B. Rawlings, Model predictive control with linear models, AIChE Journal 39 (2) (1993) 262-287. 
[40] G. Pannocchia, J. B. Rawlings, Disturbance models for offset-free model-predictive control, AIChE Journal 49 (2) (2003) 426-437.

[41] K. R. Muske, T. A. Badgwell, Disturbance modeling for offset-free linear model predictive control, Journal of Process Control 12 (5) (2002) 617-632.

[42] J. B. Rawlings, D. Q. Mayne, Model Predictive Control: Theory and Design, Nob Hill Publishing, 2009. 